\title{
Convergence Analysis of Iterative Learning Control for Two Classes of 2-D Linear Discrete Fornasini-Marchesini Model
}

\author{
Kai Wan \\ School of Electronic Information and Electrical Engineering, Huizhou University, Huizhou 516007, China \\ Correspondence should be addressed to Kai Wan; wankai606815@163.com
}

Received 2 July 2020; Revised 23 August 2020; Accepted 1 September 2020; Published 14 September 2020

Academic Editor: Guangchen Zhang

Copyright (c) 2020 Kai Wan. This is an open access article distributed under the Creative Commons Attribution License, which permits unrestricted use, distribution, and reproduction in any medium, provided the original work is properly cited.

\begin{abstract}
This paper first investigates convergent property of two iterative learning control (ILC) laws for two kinds of two-dimensional linear discrete systems described by the first Fornasini-Marchesini model (2-D LDFFM with a direct transmission from inputs to outputs and 2-D LDFFM with input delay). Different from existing ILC results for 2-D LDFFM, this paper provides convergence analysis in a three-dimensional (3-D) framework. By using row scanning approach (RSA) or column scanning approach (CSA), it is theoretically proved no matter which method is adopted, perfect tracking on the desired reference surface is accomplished. In addition, linear matrix inequality (LMI) technique is utilized to computer the learning gain of the ILC controller. The effectiveness and feasibility of the designed ILC law are illustrated through numerical simulation on a practical thermal process.
\end{abstract}

\section{Introduction}

In practical industrial applications for two-dimensional (2-D) dynamical systems, for example, in form-closure grasps, the immobilized manipulation of 2-D serial chains could be regarded as a repetitive control problem [1]. Also, mold processing and material manufacturing usually require repeated operations of the controlled processing units to obtain a high-precision reference surface [2]. With regard to this kind of repetitive tracking problem, the traditional control methods in infinite coordinate domain, such as $H_{\infty}$ control [3], stochastic stability analysis [4], and sliding mode control [5], are difficult to be used because the precise model information is known in advance. Clearly, for the repetitively tracking cases mentioned above, the traditional tracking control approaches for 2-D dynamical systems in infinite coordinate domain are difficult to be applicable. Clearly, for the repetitively tracking cases mentioned above, the traditional tracking control approaches for 2-D dynamical systems in infinite coordinate domain are difficult to be applicable.

To the best of our knowledge, iterative learning control (ILC), as a data-driven and unsupervised control approach, does not require accurate knowledge of the controlled system, which makes ILC be widely prevalent in practical applications. A large number of ILC research results reported in the past few decades have fundamentally designed for one-dimensional (1-D) dynamical systems [6-14], only very few results involved in 2-D dynamical systems [15-22], which concentrate on mainly 2-D linear discrete first Fornasini-Marchesini model (2-D LDFFM). An optimal ILC algorithm was proposed in [16], such that the ILC tracking error converges to zero monotonically. Unfortunately, the system parameters of 2-D LDFFM are known. In [15], five ILC algorithms were proposed, the effectiveness and feasibility of which is verified through only numerical example. Afterwards, by using the column scanning approach (CSA), the iterative process of 2-D LDFFM is transformed into a iterative process of $1 \mathrm{D}$ systems; rigorously, theoretical proof on convergence and robustness of a two-gain ILC law is provided in [17]. Also, using the CSA as [18], to track a class of nonrepetitive reference surface described by a high-order internal model operator (HOIM), two HOIM-based ILC laws were, respectively, investigated in [23] for 2-D LDFFM by using 2-D HOIM-based linear inequality theory, but the ultimate ILC tracking error can only converge to a bounded range. To this end, adaptive ILC approach was proposed in $[21,22]$ to identify all unknown system parameters of 2-D 
LDFFM, and the ILC result of perfect tracking on iterationvarying reference surface can be obtained. Unfortunately, the gain matrix in 2-D LDFFM is required to be positive definite, such that the proposed adaptive ILC algorithm, in practical applications, is greatly restricted. Notably, the aforementioned ILC results have concerned mainly on 2-D LDFFM without input delay.

Time delay, recognized as one of the main sources for poor performance or even instability of control systems, is frequently encountered in the signal transmission process of engineering and biological systems [24]. Over the past few years, with respect to 2-D systems with time delay on horizontal direction and vertical direction, some initial results have been reported on the states estimation [25], $H_{\infty}$ control and filtering [26,27], and nonfragile robust optimal guaranteed cost control [28]. For instance, the authors in [23] have investigated the robust state estimation problem for 2-D linear discrete systems with state delay, and a robust $H_{\infty}$ filter for 2-D discrete systems with time delays has been designed in [23]. A delay-dependent $H_{\infty}$ controller for 2-D discrete state delay systems was designed in [27]. Nonfragile robust optimal guaranteed cost control subject to both state delay and input delay is discussed in [28]. However, no works have considered the ILC tracking problem for 2-D linear discrete systems with input delay, which motivates our current study.

This paper first investigates the convergence property of two ILC laws for 2-D LDFFM with a direct transmission from inputs to outputs and 2-D LDFFM with input delay to track the desired reference surface, respectively. The 2-D LDFFM is very typical, and many practical 2-D engineering systems can be represented as the form of 2-D LDFFM $[29,30]$. Under a 3-D framework, a 3-D dynamical process is converted into a 2-D dynamical process by using CSA or row scanning approach (RSA), and it is theoretically proved no matter which method is used; perfect tracking on the desired reference surface is fulfilled. Additionally, this paper theoretically provides a linear matrix inequality (LMI) technique to computer the learning gain matrix of the ILC law. The main contributions of this paper are summarized as follows:

(1) All of the existing ILC results for 2-D dynamical systems have not considered input delay. To the best of our knowledge, it is the first time to investigate ILC algorithms for 2-D LDFFM with a direct transmission from inputs to outputs and for 2-D LDFFM with input delay.

(2) Compared with the adaptive ILC algorithm for 2-D LDFFM in [21, 22], two ILC algorithms proposed in this paper have no restriction on the numbers of system inputs and outputs.

(3) Different from the existing ILC work for 2-D LDFFM $[17,18]$, a $3-D$ framework learning mechanism is presented in this paper and can reveal the dynamical behavior of the 2-D LDFFM in the horizontal dynamical direction, vertical dynamical direction, and iteration direction.
(4) It is theoretically proved that RSA or CSA can guarantee perfect tracking on the desired reference surface.

(5) Compared with the literature [20], this paper has addressed the ILC issue on nonidentical boundary states. In addition, this paper successfully avoids strong Assumption 2.2 on desired reference surface and Assumption 2.3 on the system matrix, and LMI technique is utilized to computer the learning gain of the ILC controller.

The rest of this paper is arranged as follows: Section 2 presents problem formulation and some preliminaries. Sections 3 and 4 provide convergence analysis of a symmetrical P-type ILC law and extend to 2-D LDFFM with input delay, respectively. Two simulation examples are introduced in Section 5. Finally, a conclusion on this article is drawn in Section 6. Notations: $A \succ 0(A \prec 0)$ denotes positivedefinite (negative-definite) matrix. $\{i\}_{m_{1}}^{m_{2}}$ represents $i \in\left\{m_{1}, m_{1}+1, m_{1}+2, \ldots, m_{2}\right\}$ with positive integer $m_{2}$, and $\{i\}_{0}^{\infty}$ represents $i \in\{0,1,2, \ldots\} . I_{m}$ denotes identity matrix with dimension $m \times m . \rho(\cdot)$ represents spectral radius of matrix.

\section{Problem Formulation and Some Preliminaries}

Consider the following 2-D LDFFM with a direct transmission from inputs to outputs [23], executing designated tracking tasks repetitively over dynamical region $\left\{n_{1}\right\}_{0}^{N_{1}}$ and $\left\{n_{2}\right\}_{0}^{N_{2}}$ :

$$
\left\{\begin{array}{l}
x_{k}\left(n_{1}+1, n_{2}+1\right)=A_{1} x_{k}\left(n_{1}+1, n_{2}\right)+A_{2} x_{k}\left(n_{1}, n_{2}\right) \\
\quad+A_{3}\left(n_{1}, n_{2}+1\right)+B u_{k}\left(n_{1}, n_{2}\right), \\
y_{k}\left(n_{1}, n_{2}\right)=C x_{k}\left(n_{1}, n_{2}\right)+D u_{k}\left(n_{1}, n_{2}\right),
\end{array}\right.
$$

where $u_{k}\left(n_{1}, n_{2}\right) \in R^{m}, x_{k}\left(n_{1}, n_{2}\right) \in R^{p}$, and $y_{k}\left(n_{1}, n_{2}\right) \in R^{l}$ represent, respectively, control input, system state, and control output; $A_{1}, A_{2}, A_{3}, B, C$, and $D$ are real matrices with appropriate dimensions. $\{k\}_{0}^{\infty}$ denotes the $k$-th repetitive number (or iteration number) of 2-D LDFFM (1); $n_{1}$ and $n_{2}$ are, respectively, discrete indexes along the horizontal dynamical direction and vertical dynamical direction. The independent indexes $n_{1}$ and $n_{2}$ in practical 2-D LDFFM, i.e., chemical reactors, heater exchangers, and pipe furnaces, usually represent space locations and time instants, respectively [31]. As $D=0,2-\mathrm{D}$ LDFFM (1) has been investigated in $[17,18]$.

For $\left\{n_{1}\right\}_{0}^{N_{1}}$ and $\left\{n_{2}\right\}_{0}^{N_{2}}$, let the desired trajectory surface and the corresponding tracking error at $k$ th iteration, respectively, be denoted as $y_{r}\left(n_{1}, n_{2}\right)$ and $e_{k}\left(n_{1}, n_{2}\right)$, and the control objective of ILC for 2-D LDFFM is to update the control input $u_{k}\left(n_{1}, n_{2}\right)$ iteratively, such that the system output $y_{k}\left(n_{1}, n_{2}\right)$ converges to desired reference surface $y_{r}\left(n_{1}, n_{2}\right)$ asymptotically, i.e.,

$$
\lim _{k \longrightarrow+\infty} y_{k}\left(n_{1}, n_{2}\right)=y_{r}\left(n_{1}, n_{2}\right), \quad\left\{n_{1}\right\}_{1}^{N_{1}},\left\{n_{2}\right\}_{1}^{N_{2}} .
$$


For the convenience of discussing the ILC problem for 2D LDFFM, the property on a block matrix, assumptions, and Lemma is provided as follows.

Property 1. (see [32]). Give the following block matrix $H$ with a nonsingular matrix $H_{1} \in R^{m \times m}$ :

$$
H=\left[\begin{array}{ccccc}
H_{1} & 0 & 0 & \cdots & 0 \\
-H_{2} & H_{1} & 0 & \cdots & \vdots \\
0 & -H_{2} & H_{1} & \ddots & 0 \\
\vdots & \ddots & \ddots & \ddots & 0 \\
0 & \cdots & 0 & -H_{2} & H_{1}
\end{array}\right] \in R^{m N \times m N}
$$

and the inverse matrix of which is given as

$$
H^{-1}=\left[\begin{array}{ccccc}
\bar{H}_{1} & 0 & 0 & \cdots & 0 \\
\bar{H}_{2} & \bar{H}_{1} & 0 & \cdots & \vdots \\
\bar{H}_{3} & \bar{H}_{2} & \bar{H}_{1} & \ddots & 0 \\
\vdots & \ddots & \ddots & \ddots & 0 \\
\bar{H}_{N} & \cdots & \bar{H}_{3} & \bar{H}_{2} & \bar{H}_{1}
\end{array}\right] \in R^{m N \times m N},
$$

with

$$
\left\{\begin{array}{l}
\bar{H}_{1}=H_{1}^{-1} \\
\bar{H}_{2}=H_{1}^{-1} H_{2} H_{1}^{-1} \\
\bar{H}_{3}=\left(H_{1}^{-1} H_{2}\right)^{2} H_{1}^{-1} \\
\vdots \\
\bar{H}_{N}=\left(H_{1}^{-1} H_{2}\right)^{N-1} H_{1}^{-1} .
\end{array}\right.
$$

In particular, as $H_{1}=I_{m}, H^{-1}$ becomes

$$
H^{-1}=\left[\begin{array}{ccccc}
I_{m} & 0 & 0 & \cdots & 0 \\
H_{2} & I_{m} & 0 & \cdots & \vdots \\
H_{2}^{2} & H_{2} & I_{m} & \ddots & 0 \\
\vdots & \ddots & \ddots & \ddots & 0 \\
H_{2}^{N-1} & \cdots & H_{2}^{2} & H_{2} & I_{m}
\end{array}\right] .
$$

Property 2 (see [32]). For $H^{-1}$ in Property 1, according to matrix theory, there is $\rho\left(H^{-1}\right)=\rho\left(\bar{H}_{1}\right)$, which is irrelevant to $\bar{H}_{2}, \bar{H}_{3}, \ldots$, and $\bar{H}_{N}$.

Remark 1. Properties 1 and 2 are often used in the ILC convergence analysis for 2-D linear discrete dynamical systems to track reference surface described by a high-order internal model or varying uniformly between $(k+1)$-th iteration and $k$-th iteration [18]. Certainly, Properties 1 and 2 are also suitable to track repetitive reference surface considered in this paper.

Assumption 1. The matrix $D$ is full-row rank.

Assumption 2. The boundary states $x_{k}\left(0, n_{2}\right)$ and $x_{k}\left(n_{1}, 0\right)$ of 2-D LDFFM (1) are assumed to satisfy $x_{k}\left(0, n_{2}\right)=x_{0}\left(0, n_{2}\right), \quad\left\{n_{2}\right\}_{1}^{N_{2}}$ and $x_{k}\left(n_{1}, 0\right)=x_{0}\left(n_{1}, 0\right)$,
$\left\{n_{1}\right\}_{0}^{N_{1}}$, where $x_{0}\left(0, n_{2}\right)$ and $x_{0}\left(n_{1}, 0\right)$ are iteration-invariant functions with respective to $n_{2}$ and $n_{1}$.

Lemma 1. Consider the following 3-D linear discrete system for $\left\{n_{1}\right\}_{0}^{N_{1}},\left\{n_{2}\right\}_{0}^{N_{2}}$, and $\{k\}_{0}^{\infty}$ :

$$
\theta_{k+1}\left(n_{1}, n_{2}\right)=\alpha_{1} \theta_{k}\left(n_{1}, n_{2}\right)+\alpha_{2} \delta_{k}\left(n_{1}, n_{2}\right),
$$

where $\theta_{k}\left(n_{1}, n_{2}\right) \in R^{L_{1}}$ and $\delta_{k}\left(n_{1}, n_{2}\right) \in R^{L_{2}}$, respectively, are state and control input; $\alpha_{1}$ and $\alpha_{2}$ denote real matrices. Suppose the boundary state $\theta_{0}\left(n_{1}, n_{2}\right)=f\left(n_{1}, n_{2}\right)$, where $f\left(n_{1}, n_{2}\right)$ is a bounded vector function. When $\lim _{k \rightarrow+\infty} \delta_{k}\left(n_{1}, n_{2}\right)=0$, if $\rho\left(\alpha_{1}\right)<1$ is satisfied, then the 3-D system is asymptotically stable along the dynamical direction $k$, i.e.,

$$
\lim _{k \longrightarrow+\infty} \theta_{k}\left(n_{1}, n_{2}\right)=0, \quad\left\{n_{1}\right\}_{0}^{N_{1}},\left\{n_{2}\right\}_{0}^{N_{2}} .
$$

In particular, when $\delta_{k}\left(n_{1}, n_{2}\right)=0$, for $\left\{n_{1}\right\}_{0}^{N_{1}}$ and $\left\{n_{2}\right\}_{0}^{N_{2}}$, $\lim _{k \longrightarrow+\infty} \theta_{k}\left(n_{1}, n_{2}\right)=0$ can still be obtained for $\left\{n_{1}\right\}_{0}^{N_{1}}$ and $\left\{n_{2}\right\}_{0}^{N_{2}}$. The proof process of Lemma 1 is shown in Appendix.

\section{Convergence Analysis of a Symmetrical P-Type ILC Law}

The following symmetrical P-type ILC law is applied to 2-D LDFFM (1) for $\left\{n_{1}\right\}_{0}^{N_{1}}$ and $\left\{n_{2}\right\}_{0}^{N_{2}}$ :

$$
u_{k+1}\left(n_{1}, n_{2}\right)=u_{k}\left(n_{1}, n_{2}\right)+\Gamma e_{k}\left(n_{1}, n_{2}\right),
$$

where $e_{k}\left(n_{1}, n_{2}\right)=y_{r}\left(n_{1}, n_{2}\right)-y_{k}\left(n_{1}, n_{2}\right)$. The learning gain $\Gamma \in R^{m \times l}$ is to be designed.

Remark 2. It is worth noting that 2-D LDFFM (1) is a zero relative degree system; the proposed ILC law (9) is extended on the existing ILC work for 1D linear discrete dynamical systems with zero relative degree [33]. Additionally, 2-D LDFFM (1) and the ILC law (9) are symmetrical on the discrete indexes $\left\{n_{1}\right\}_{0}^{N_{1}}$ and $\left\{n_{2}\right\}_{0}^{N_{2}}$; therefore, row scanning approach (RSA) or column scanning approach (CSA) can guarantee the same ILC tracking result, which is demonstrated in Theorem 1 and Appendix.

Theorem 1. Consider the 2-D LDFFM with a direct transmission from inputs to outputs (1) under Assumptions 1 and 2 and use the symmetrical P-type ILC law (9). If the learning gain $\Gamma$ satisfies

$$
\rho\left(I_{l}-D \Gamma\right)<1
$$

then the tracking error $e_{k}\left(n_{1}, n_{2}\right)$ converges to zero, i.e.,

$$
\lim _{k \longrightarrow+\infty} e_{k}\left(n_{1}, n_{2}\right)=0, \quad\left\{n_{1}\right\}_{0}^{N_{1}},\left\{n_{2}\right\}_{0}^{N_{2}} .
$$

Proof 1. For $\left\{n_{1}\right\}_{0}^{N_{1}-1}$ and $\left\{n_{2}\right\}_{0}^{N_{2}-1}$, let

$$
\begin{aligned}
& \delta x_{k}\left(n_{1}, n_{2}\right)=x_{k+1}\left(n_{1}, n_{2}\right)-x_{k}\left(n_{1}, n_{2}\right), \\
& \delta u_{k}\left(n_{1}, n_{2}\right)=u_{k+1}\left(n_{1}, n_{2}\right)-u_{k}\left(n_{1}, n_{2}\right) .
\end{aligned}
$$


Using (1), for $\left\{n_{1}\right\}_{0}^{N_{1}-1}$ and $\left\{n_{2}\right\}_{0}^{N_{2}-1}$, there is

$$
\begin{aligned}
\delta x_{k}\left(n_{1}+1, n_{2}+1\right)= & x_{k+1}\left(n_{1}+1, n_{2}+1\right) \\
& -x_{k}\left(n_{1}+1, n_{2}+1\right) \\
= & A_{1} \delta x_{k}\left(n_{1}+1, n_{2}\right)+A_{2} \delta x_{k}\left(n_{1}, n_{2}\right) \\
& +A_{3} \delta x_{k}\left(n_{1}, n_{2}+1\right)+B \delta u_{k}\left(n_{1}, n_{2}\right) .
\end{aligned}
$$

Applying the ILC law (9) into (13), it generates

$$
\begin{aligned}
\delta x_{k}\left(n_{1}+1, n_{2}+1\right)= & A_{1} \delta x_{k}\left(n_{1}+1, n_{2}\right)+A_{2} \delta x_{k}\left(n_{1}, n_{2}\right) \\
& +A_{3} \delta x_{k}\left(n_{1}, n_{2}+1\right)+B \Gamma e_{k}\left(n_{1}, n_{2}\right) .
\end{aligned}
$$

On the contrary, according to $e_{k}\left(n_{1}, n_{2}\right)=y_{r}\left(n_{1}, n_{2}\right)-$ $y_{k}\left(n_{1}, n_{2}\right)$ and $(1)$, we have

$$
\begin{aligned}
e_{k+1}\left(n_{1}, n_{2}\right)-e_{k}\left(n_{1}, n_{2}\right)= & y_{r}\left(n_{1}, n_{2}\right)-y_{k+1}\left(n_{1}, n_{2}\right) \\
& -y_{r}\left(n_{1}, n_{2}\right)+y_{k}\left(n_{1}, n_{2}\right) \\
= & -C\left[x_{k+1}\left(n_{1}, n_{2}\right)-x_{k}\left(n_{1}, n_{2}\right)\right] \\
& -D\left[u_{k+1}\left(n_{1}, n_{2}\right)-u_{k}\left(n_{1}, n_{2}\right)\right],
\end{aligned}
$$

where $\left\{n_{1}\right\}_{0}^{N_{1}}$ and $\left\{n_{2}\right\}_{0}^{N_{2}}$. Substituting the ILC law (9) into (15), it yields

$$
\begin{aligned}
e_{k+1}\left(n_{1}, n_{2}\right)-e_{k}\left(n_{1}, n_{2}\right)= & -C\left[x_{k+1}\left(n_{1}, n_{2}\right)-x_{k}\left(n_{1}, n_{2}\right)\right] \\
& -D \Gamma e_{k}\left(n_{1}, n_{2}\right) .
\end{aligned}
$$

Rearranging (16), there is

$$
e_{k+1}\left(n_{1}, n_{2}\right)=-C \delta x_{k}\left(n_{1}, n_{2}\right)+\left(I_{l}-D \Gamma\right) e_{k}\left(n_{1}, n_{2}\right) \text {. }
$$

Let

$$
\begin{gathered}
\delta X_{k}\left(n_{2}\right)=\left[\begin{array}{llll}
\delta x_{k}^{T}\left(1, n_{2}\right) & \delta x_{k}^{T}\left(2, n_{2}\right) & \cdots & \delta x_{k}^{T}\left(N_{1}, n_{2}\right)
\end{array}\right]^{T}, \\
E_{k}\left(n_{2}\right)=\left[\begin{array}{llll}
e_{k}^{T}\left(0, n_{2}\right) & e_{k}^{T}\left(1, n_{2}\right) & \cdots & e_{k}^{T}\left(N_{1}, n_{2}\right)
\end{array}\right]^{T},
\end{gathered}
$$

where $\left\{n_{2}\right\}_{0}^{N_{2}}$. According to (18) and (19), using the CSA on horizontal dynamical direction $n_{1}$, (14) can be rewritten as

$$
\begin{aligned}
\Phi_{1} \delta X_{k}\left(n_{2}+1\right)= & \Phi_{2} \delta X_{k}\left(n_{2}\right)+\Phi_{3} E_{k}\left(n_{2}\right)+\widehat{A}_{2} \delta x_{k}\left(0, n_{2}\right) \\
& +\widehat{A}_{3} \delta x_{k}\left(0, n_{2}+1\right),
\end{aligned}
$$

where
$\Phi_{1}=\left[\begin{array}{ccccc}I_{p} & 0 & 0 & \cdots & 0 \\ -A_{3} & I_{p} & 0 & \cdots & 0 \\ 0 & -A_{3} & I_{p} & \ddots & \vdots \\ \vdots & \ddots & \ddots & \ddots & 0 \\ 0 & \cdots & 0 & -A_{3} & I_{p}\end{array}\right] \in R^{p N_{1} \times p N_{1}}$

$\Phi_{2}=\left[\begin{array}{ccccc}A_{1} & 0 & 0 & \cdots & 0 \\ A_{2} & A_{1} & 0 & \cdots & 0 \\ 0 & A_{2} & A_{1} & \ddots & \vdots \\ \vdots & \ddots & \ddots & \ddots & 0 \\ 0 & \cdots & 0 & A_{2} & A_{1}\end{array}\right] \in R^{p N_{1} \times p N_{1}}$,

$\Phi_{3}=\left[\begin{array}{ccccc}B \Gamma & 0 & 0 & \cdots & 0 \\ 0 & B \Gamma & \ddots & \ddots & \vdots \\ 0 & 0 & B \Gamma & 0 & 0 \\ \vdots & \ddots & \ddots & \ddots & 0 \\ 0 & \cdots & 0 & 0 & B \Gamma\end{array}\right] \in R^{p N_{1} \times l\left(N_{1}+1\right)}$,

$\widehat{A}_{2}=\left[\begin{array}{c}A_{2} \\ 0 \\ 0 \\ \vdots \\ 0\end{array}\right] \in R^{p N_{1} \times p}$,

$\widehat{A}_{3}=\left[\begin{array}{c}A_{3} \\ 0 \\ 0 \\ \vdots \\ 0\end{array}\right] \in R^{p N_{1} \times p}$.

Then, since $\Phi_{1}$ is a nonsingular matrix, premultiplying $\Phi_{1}^{-1}$ on both sides of (20), we get

$$
\begin{aligned}
\delta X_{k}\left(n_{2}+1\right)= & \bar{\Phi}_{1} \delta X_{k}\left(n_{2}\right)+\bar{\Phi}_{2} E_{k}\left(n_{2}\right)+\Phi_{1}^{-1} \widehat{A}_{2} \delta x_{k}\left(0, n_{2}\right) \\
& +\Phi_{1}^{-1} \widehat{A}_{3} \delta x_{k}\left(0, n_{2}+1\right),
\end{aligned}
$$

where $\bar{\Phi}_{1}=\Phi_{1}^{-1} \Phi_{2}$ and $\bar{\Phi}_{2}=\Phi_{1}^{-1} \Phi_{3}$. Similarly, (17) can also rewritten as

$$
E_{k+1}\left(n_{2}\right)=\varphi_{1} \delta X_{k}\left(n_{2}\right)+\varphi_{2} E_{k}\left(n_{2}\right)-C \delta x_{k}\left(0, n_{2}\right),
$$

where 


$$
\begin{aligned}
\varphi_{1} & =\left[\begin{array}{ccccc}
0 & 0 & 0 & \cdots & 0 \\
-C & 0 & 0 & \cdots & 0 \\
0 & -C & 0 & \ddots & \vdots \\
\vdots & \ddots & \ddots & \ddots & 0 \\
0 & \cdots & 0 & -C & 0
\end{array}\right] \in R^{l\left(N_{1}+1\right) \times p N_{1}}, \\
\varphi_{2} & =\left[\begin{array}{cccc}
I_{l}-D \Gamma & 0 & \cdots & 0 \\
0 & I_{l}-D \Gamma & \ddots & \vdots \\
\vdots & \ddots & \ddots & 0 \\
0 & \cdots & 0 & I_{l}-D \Gamma
\end{array}\right] \in R^{l\left(N_{1}+1\right) \times l\left(N_{1}+1\right)}, \\
\widehat{C} & =\left[\begin{array}{c}
C \\
0 \\
\vdots \\
0
\end{array}\right] \in R^{l\left(N_{1}+1\right) \times p} .
\end{aligned}
$$

From Assumption 2, we obtain $\delta x_{k}\left(0, n_{2}\right)=$ $x_{k+1}\left(0, n_{2}\right)-x_{k}\left(0, n_{2}\right)=0$ for $\left\{n_{2}\right\}_{0}^{N_{2}}$. Thus, (22) and (23) can be reformulated as

$$
\left\{\begin{array}{l}
\delta X_{k}\left(n_{2}+1\right)=\bar{\Phi}_{1} \delta X_{k}\left(n_{2}\right)+\bar{\Phi}_{2} E_{k}\left(n_{2}\right), \\
E_{k+1}\left(n_{2}\right)=\varphi_{1} \delta X_{k}\left(n_{2}\right)+\varphi_{2} E_{k}\left(n_{2}\right),
\end{array}\right.
$$

where $\left\{n_{2}\right\}_{0}^{N_{2}-1}$. In (25), based on Assumption 2, we deduce

$$
\delta X_{k}(0)=\left[\begin{array}{lllll}
\delta x_{k}^{T}(1,0) & \delta x_{k}^{T}(2,0) & \cdots & \delta x_{k}^{T}\left(N_{1}, 0\right)
\end{array}\right]^{T}=0,
$$

and $E_{0}\left(n_{2}\right)$ is bounded for $\left\{n_{2}\right\}_{0}^{N_{2}-1}$. Using Lemma 4 in [18], if $\rho\left(\varphi_{2}\right)<1$ (equivalently, $\rho\left(I_{l}-D \Gamma\right)<1$, see Property 2), there is

$$
\left\{\begin{array}{l}
\lim _{k \longrightarrow+\infty} \delta X_{k}\left(n_{2}\right)=0,\left\{n_{2}\right\}_{1}^{N_{2}}, \\
\lim _{k \longrightarrow+\infty} E_{k}\left(n_{2}\right)=0,\left\{n_{2}\right\}_{0}^{N_{2}-1} .
\end{array}\right.
$$

Additionally, taking $n_{2}=N_{2}$ in (17), we have

$$
e_{k+1}\left(n_{1}, N_{2}\right)=-C \delta x_{k}\left(n_{1}, N_{2}\right)+\left(I_{l}-D \Gamma\right) e_{k}\left(n_{1}, N_{2}\right) \text {, }
$$

where $\left\{n_{1}\right\}_{0}^{N_{1}}$. From (27) and $\delta x_{k}\left(0, N_{2}\right)=0$, we obtain $\lim _{k \longrightarrow+\infty} \delta x_{k}\left(n_{1}, N_{2}\right)=0,\left\{n_{1}\right\}_{0}^{N_{1}}$. Using Lemma 1, if $\rho\left(I_{l}-\right.$ $D \Gamma)<1$ is satisfied, there is

$$
\lim _{k \longrightarrow+\infty} e_{k}\left(n_{1}, N_{2}\right)=0, \quad\left\{n_{1}\right\}_{0}^{N_{1}} .
$$

From (27) and (29) and the definition on $E_{k}\left(n_{2}\right)$ in (19), it yields

$$
\lim _{k \longrightarrow+\infty} e_{k}\left(n_{1}, n_{2}\right)=0, \quad\left\{n_{1}\right\}_{0}^{N_{1}},\left\{n_{2}\right\}_{0}^{N_{2}} .
$$

Theorem 1 is completed.

Remark 3. The convergence condition $\rho\left(I_{l}-D \Gamma\right)<1$ in Theorem 1 only depends on the matrix $D$ and is irrelevant to $A_{1}, A_{2}, A_{3}, C$, and $B$, which is in accordance with the ILC convergence condition of the following 1D linear discrete iterative system to track repetitive reference trajectory [33]:

$$
\left\{\begin{array}{l}
x_{k}(t+1)=A x_{k}(t)+B u_{k}(t) \\
y_{k}(t)=C x_{k}(t)+D u_{k}(t)
\end{array}\right.
$$

under a P-type ILC law $u_{k+1}(t)=u_{k}(t)+\Gamma e_{k}(t)$ and initial iterative state $x_{k}(t)=x_{0}(t)$.

Remark 4. Taking advantage of the estimated information on $D$, the learning gain matrix $\Gamma$ of $\rho\left(I_{l}-D \Gamma\right)<1$ can be determined by using LMI technique. For the following $1 \mathrm{D}$ linear discrete dynamical system described by $I_{l}-D \Gamma$,

$$
x(k+1)=\left(I_{l}-D \Gamma\right) x(k), \quad\{k\}_{0}^{\infty} .
$$

Asymptotically stable condition $\rho\left(I_{l}-D \Gamma\right)<1$ is satisfied if and only if there exists a $Q>0$ such that

$$
\left(I_{l}-D \Gamma\right)^{T} Q\left(I_{l}-D \Gamma\right)-Q \prec 0 .
$$

Using Schur complement theorem [34], it yields

$$
\left[\begin{array}{cc}
-Q^{-1} & I_{l}-D \Gamma \\
I_{l}-\Gamma^{T} D^{T} & -Q
\end{array}\right]<0 .
$$

Pre- and postmultiplying by $\operatorname{diag}\left\{I_{l}, Q^{-1}\right\}$ on both sides of (34), we have

$$
\left[\begin{array}{cc}
-Q^{-1} & Q^{-1}-D \Gamma Q^{-1} \\
Q^{-1}-Q^{-1} \Gamma^{T} D^{T} & -Q^{-1}
\end{array}\right]<0 .
$$

Therefore, $\rho\left(I_{l}-D \Gamma\right)<1$ is equivalent to solving the following LMI: there exists positive-definite matrix $Q \in R^{l \times l}$ and matrix $X \in R^{m \times l}$ such that

$$
\left[\begin{array}{cc}
-Q^{-1} & Q^{-1}-D X \\
Q^{-1}-X^{T} D^{T} & -Q^{-1}
\end{array}\right] \prec 0,
$$

with $X=\Gamma Q^{-1}$. Therefore, the selection problem of the learning gain matrix $\Gamma$ is transformed into the feasibility problem on solving LMI (36).

Remark 5. Theorem 1 employs the CSA on dynamical direction $n_{1}$ to obtain ILC result under Assumption 2. As the RSA on dynamical direction $n_{2}$ is applied to 2-D LDFFM (1) and the ILC law (9), we can still acquire the same ILC result with Theorem 1 due to the symmetrical characteristics on $n_{1}$ and $n_{2}$ in 2-D LDFFM (1) and the ILC law (9), and see Appendix.

\section{Extension to 2-D LDFFM with Input Delay}

In this section, under Assumption 2, we investigate the ILC issues for 2-D LDFFM with input delay [23], which is described in the following for $\left\{n_{1}\right\}_{0}^{N_{1}-1}$ and $\left\{n_{2}\right\}_{0}^{N_{2}-1}$ :

$$
\left\{\begin{array}{l}
x_{k}\left(n_{1}+1, n_{2}+1\right)=A_{1} x_{k}\left(n_{1}+1, n_{2}\right)+A_{2} x_{k}\left(n_{1}, n_{2}\right) \\
\quad+A_{3} x_{k}\left(n_{1}, n_{2}+1\right)+B u_{k}\left(n_{1}-\tau_{1}, n_{2}-\tau_{2}\right), \\
y_{k}\left(n_{1}, n_{2}\right)=C x_{k}\left(n_{1}, n_{2}\right),
\end{array}\right.
$$


where $\tau_{1}$ and $\tau_{2}$ are two positive integers. $x_{k}\left(n_{1}, n_{2}\right)$, $u_{k}\left(n_{1}, n_{2}\right)$, and $y_{k}\left(n_{1}, n_{2}\right)$ have been described in (1).

Remark 6. It is worthwhile noting that ILC tracking issues for 2-D LDFFM with $\tau_{1}=0$ and $\tau_{2}=0$ in (37) have been investigated in $[17,18]$. It is the first time that ILC issues for 2-D LDFFM with input delay are investigated in this paper.

Assumption 3. The matrix CB is full-row rank.

Next, we discuss the convergence property of the ILC law for 2-D LDFFM with input delay (37) under Assumptions 2 and 3 , and there is the following Theorem 2.

Theorem 2. Consider the 2-D LDFFM with input delay (37) under Assumptions 2 and 3 and use the following ILC law for $\left\{n_{1}\right\}_{-\tau_{1}}^{N_{1}-\tau_{1}-1}$ and $\left\{n_{2}\right\}_{-\tau_{2}}^{N_{2}-\tau_{2}-1}$ :

$$
u_{k+1}\left(n_{1}, n_{2}\right)=u_{k}\left(n_{1}, n_{2}\right)+\Gamma e_{k}\left(n_{1}+\tau_{1}+1, n_{2}+\tau_{2}+1\right) .
$$

If the learning gain $\Gamma$ satisfies

$$
\rho\left(I_{l}-C B \Gamma\right)<1,
$$

then the tracking error $e_{k}\left(n_{1}, n_{2}\right)$ converges to zero, i.e.,

$$
\lim _{k \longrightarrow+\infty} e_{k}\left(n_{1}, n_{2}\right)=0, \quad\left\{n_{1}\right\}_{1}^{N_{1}},\left\{n_{2}\right\}_{1}^{N_{2}} .
$$

Proof 2. For $\left\{n_{1}\right\}_{0}^{N_{1}}$ and $\left\{n_{2}\right\}_{0}^{N_{2}}$, let

$$
\delta \widehat{x}_{k}\left(n_{1}, n_{2}+1\right)=x_{k+1}\left(n_{1}, n_{2}\right)-x_{k}\left(n_{1}, n_{2}\right) .
$$

From (37), there is

$$
\begin{aligned}
\delta \widehat{x}_{k}\left(n_{1}+1, n_{2}+1\right)= & x_{k+1}\left(n_{1}+1, n_{2}\right)-x_{k}\left(n_{1}+1, n_{2}\right) \\
= & A_{1} \delta \widehat{x}_{k}\left(n_{1}+1, n_{2}\right)+A_{2} \delta \widehat{x}_{k}\left(n_{1}, n_{2}\right) \\
& +B\left[u_{k+1}\left(n_{1}-\tau_{1}, n_{2}-\tau_{2}-1\right)\right. \\
& \left.-u_{k}\left(n_{1}-\tau_{1}, n_{2}-\tau_{2}-1\right)\right],
\end{aligned}
$$

where $\left\{n_{1}\right\}_{0}^{N_{1}-1}$ and $\left\{n_{2}\right\}_{1}^{N_{2}}$. Inserting the ILC law (38) into (42), it generates

$$
\begin{aligned}
\delta \widehat{x}_{k}\left(n_{1}+1, n_{2}+1\right)= & A_{1} \delta \widehat{x}_{k}\left(n_{1}+1, n_{2}\right)+A_{2} \delta \widehat{x}_{k}\left(n_{1}, n_{2}\right) \\
& +A_{3} \delta \widehat{x}_{k}\left(n_{1}, n_{2}+1\right)+B \Gamma e_{k}\left(n_{1}+1, n_{2}\right) .
\end{aligned}
$$

On the contrary, according to $e_{k}\left(n_{1}, n_{2}\right)=y_{r}\left(n_{1}, n_{2}\right)-$ $y_{k}\left(n_{1}, n_{2}\right)$ and using (37), we have

$$
\begin{aligned}
e_{k+1} & \left(n_{1}+1, n_{2}\right)-e_{k}\left(n_{1}+1, n_{2}\right) \\
= & y_{r}\left(n_{1}+1, n_{2}\right)-y_{k+1}\left(n_{1}+1, n_{2}\right)-y_{r}\left(n_{1}+1, n_{2}\right) \\
& +y_{k}\left(n_{1}+1, n_{2}\right) \\
= & -C\left[x_{k+1}\left(n_{1}+1, n_{2}\right)-x_{k}\left(n_{1}+1, n_{2}\right)\right] \\
= & -C \delta \widehat{x}_{k}\left(n_{1}+1, n_{2}+1\right),
\end{aligned}
$$

where $\left\{n_{1}\right\}_{0}^{N_{1}-1}$ and $\left\{n_{2}\right\}_{0}^{N_{2}}$. Let

$$
\begin{aligned}
\delta \widehat{X}_{k}\left(n_{2}\right) & =\left[\begin{array}{llll}
\delta \widehat{x}_{k}^{T}\left(1, n_{2}\right) & \delta \widehat{x}_{k}^{T}\left(2, n_{2}\right) & \cdots & \delta \widehat{x}_{k}^{T}\left(N_{1}, n_{2}\right)
\end{array}\right]^{T} \\
E_{k}\left(n_{2}\right) & =\left[\begin{array}{llll}
e_{k}^{T}\left(1, n_{2}\right) & e_{k}^{T}\left(2, n_{2}\right) & \cdots & e_{k}^{T}\left(N_{1}, n_{2}\right)
\end{array}\right]^{T},
\end{aligned}
$$

where $\left\{n_{2}\right\}_{1}^{N_{2}}$. From (45) and (46), (43) can be rewritten as

$$
\begin{aligned}
\phi_{1} \delta \widehat{X}_{k}\left(n_{2}+1\right)= & \phi_{2} \delta \widehat{X}_{k}\left(n_{2}\right)+\phi_{3} E_{k}\left(n_{2}\right)+\widehat{A}_{2} \delta \widehat{x}_{k}\left(0, n_{2}\right) \\
& +\widehat{A}_{3} \delta \widehat{x}_{k}\left(0, n_{2}+1\right),
\end{aligned}
$$

where

$$
\phi_{1}=\left[\begin{array}{ccccc}
I_{p} & 0 & 0 & \cdots & 0 \\
-A_{3} & I_{p} & 0 & \cdots & 0 \\
0 & -A_{3} & I_{p} & \ddots & \vdots \\
\vdots & \ddots & \ddots & \ddots & 0 \\
0 & \cdots & 0 & -A_{3} & I_{p}
\end{array}\right] \in R^{p N_{1} \times p N_{1}}
$$$$
\phi_{2}=\left[\begin{array}{ccccc}
A_{1} & 0 & 0 & \cdots & 0 \\
0 & A_{1} & 0 & \cdots & 0 \\
0 & 0 & A_{1} & \ddots & \vdots \\
\vdots & \ddots & \ddots & \ddots & 0 \\
0 & \cdots & 0 & 0 & A_{1}
\end{array}\right] \in R^{p N_{1} \times p N_{1}},
$$

$$
\phi_{3}=\left[\begin{array}{ccccc}
B \Gamma & 0 & 0 & \cdots & 0 \\
0 & B \Gamma & 0 & \cdots & 0 \\
0 & 0 & B \Gamma & \ddots & \vdots \\
\vdots & \ddots & \ddots & \ddots & 0 \\
0 & \cdots & 0 & 0 & B \Gamma
\end{array}\right] \in R^{p N_{1} \times l N_{1}},
$$

and $\widehat{A}_{2}$ and $\widehat{A}_{3}$ have been described in (20). Similarly, (44) can also be reformulated as

$$
E_{k+1}\left(n_{2}\right)=\psi_{1} \delta \widehat{X}_{k}\left(n_{2}+1\right)+\psi_{2} E_{k}\left(n_{2}\right),
$$

where

$$
\begin{aligned}
& \psi_{1}=\left[\begin{array}{ccccc}
-C & 0 & 0 & \cdots & 0 \\
0 & -C & 0 & \ddots & \vdots \\
0 & 0 & -C & \ddots & 0 \\
\vdots & \ddots & \ddots & \ddots & 0 \\
0 & \cdots & 0 & 0 & -C
\end{array}\right] \in R^{l N_{1} \times p N_{1}}, \\
& \psi_{2}=\left[\begin{array}{ccccc}
I_{l} & 0 & 0 & \cdots & 0 \\
0 & I_{l} & 0 & \cdots & 0 \\
0 & 0 & I_{l} & \ddots & \vdots \\
\vdots & \ddots & \ddots & \ddots & 0 \\
0 & \cdots & 0 & 0 & I_{l}
\end{array}\right] \in R^{l N_{1} \times l N_{1}} .
\end{aligned}
$$

Then, since $\phi_{1}$ is a nonsingular matrix, premultiplying $\phi_{1}^{-1}$ on both sides of (47), we get 


$$
\begin{aligned}
\delta \widehat{X}_{k}\left(n_{2}+1\right)= & \bar{\phi}_{1} \delta \widehat{X}_{k}\left(n_{2}\right)+\bar{\phi}_{2} E_{k}\left(n_{2}\right)+\phi_{1}^{-1} \widehat{A}_{2} \delta \widehat{x}_{k}\left(0, n_{2}\right) \\
& +\phi_{1}^{-1} \widehat{A}_{3} \delta \widehat{x}_{k}\left(0, n_{2}+1\right)
\end{aligned}
$$

where $\bar{\phi}_{1}=\phi_{1}^{-1} \phi_{2}$ and $\bar{\phi}_{2}=\phi_{1}^{-1} \phi_{3}$. Substituting (51) into (49), it yields

$$
\begin{aligned}
E_{k+1}\left(n_{2}\right)= & \psi_{1} \bar{\phi}_{1} \delta \widehat{X}_{k}\left(n_{2}\right)+\left(\psi_{1} \bar{\phi}_{2}+\psi_{2}\right) E_{k}\left(n_{2}\right) \\
& +\psi_{1} \phi_{1}^{-1} \widehat{A}_{2} \delta \widehat{x}_{k}\left(0, n_{2}\right)+\psi_{1} \phi_{1}^{-1} \widehat{A}_{3} \delta \widehat{x}_{k}\left(0, n_{2}+1\right),
\end{aligned}
$$

where $\left\{n_{2}\right\}_{1}^{N_{2}}$. From Assumption 2, we obtain $\delta \widehat{x}_{k}\left(0, n_{2}+\right.$ $1)=x_{k+1}\left(0, n_{2}\right)-x_{k}\left(0, n_{2}\right)=0$ for $\left\{n_{2}\right\}_{0}^{N_{2}}$. Thus, for $\left\{n_{2}\right\}_{1}^{N_{2}}$, (51) and (52) can be represented as

$$
\left\{\begin{array}{l}
\delta \widehat{X}_{k}\left(n_{2}+1\right)=\bar{\phi}_{1} \delta \widehat{X}_{k}\left(n_{2}\right)+\bar{\phi}_{2} E_{k}\left(n_{2}\right), \\
E_{k+1}\left(n_{2}\right)=\psi_{1} \bar{\phi}_{1} \delta \widehat{X}_{k}\left(n_{2}\right)+\left(\psi_{1} \bar{\phi}_{2}+\psi_{2}\right) E_{k}\left(n_{2}\right) .
\end{array}\right.
$$

In (53), based on Assumption 2, we obtain $\delta \widehat{x}_{k}\left(n_{1}, 1\right)=x_{k+1}\left(n_{1}, 0\right)-x_{k}\left(n_{1}, 0\right)=0$. Furthermore,

$$
\delta \widehat{X}_{k}(1)=\left[\begin{array}{lllll}
\delta \hat{x}_{k}^{T}(1,1) & \delta \widehat{x}_{k}^{T}(2,1) & \cdots & \delta \widehat{x}_{k}^{T}\left(N_{1}, 1\right)
\end{array}\right]^{T}=0,
$$

and $E_{0}\left(n_{2}\right)$ is bounded for $\left\{n_{2}\right\}_{1}^{N_{2}}$ due to the boundedness property of $e_{0}\left(n_{1}, n_{2}\right)$. Using Lemma 4 in [18], if $\rho\left(\psi_{1} \bar{\phi}_{2}+\right.$ $\left.\psi_{2}\right)<1$ holds (equivalently, $\rho\left(I_{l}-\mathrm{CB} \Gamma\right)<1$, see Remark 7), there is

$$
\lim _{k \longrightarrow+\infty} E_{k}\left(n_{2}\right)=0, \quad\left\{n_{2}\right\}_{1}^{N_{2}} .
$$

From (46), we obtain

$$
\lim _{k \longrightarrow+\infty} e_{k}\left(n_{1}, n_{2}\right)=0, \quad\left\{n_{1}\right\}_{1}^{N_{2}},\left\{n_{2}\right\}_{1}^{N_{2}},
$$

Theorem 2 is completed.

Remark 7. The convergence condition $\rho\left(\psi_{1} \bar{\phi}_{2}+\psi_{2}\right)<1$ in (53) depends on a complicated matrix $\psi_{1} \bar{\phi}_{2}+\psi_{2}$; thus, there is a need to simplify it. From the definition of $\psi_{1} \bar{\phi}_{2}+\psi_{2}$ and using Property 1 , the simplifying process is shown as follows:

$$
\begin{aligned}
\psi_{1} \bar{\phi}_{2}+\psi_{2} & =\left[\begin{array}{cccc}
-C & 0 & \cdots & 0 \\
0 & -C & \ddots & \vdots \\
\vdots & \ddots & \ddots & 0 \\
0 & \cdots & 0 & -C
\end{array}\right]\left[\begin{array}{ccccc}
I_{p} & 0 & 0 & \cdots & 0 \\
-A_{3} & I_{p} & 0 & \cdots & 0 \\
0 & -A_{3} & I_{p} & \ddots & \vdots \\
\vdots & \ddots & \ddots & \ddots & 0 \\
0 & \cdots & 0 & -A_{3} & I_{p}
\end{array}\right]^{-1}\left[\begin{array}{cccc}
B \Gamma & 0 & \cdots & 0 \\
0 & B \Gamma & \ddots & \vdots \\
\vdots & \ddots & \ddots & 0 \\
0 & \cdots & 0 & B \Gamma
\end{array}\right]+\left[\begin{array}{cccc}
I_{l} & 0 & \cdots & 0 \\
0 & I_{l} & \ddots & \vdots \\
\vdots & \ddots & \ddots & 0 \\
0 & \cdots & 0 & I_{l}
\end{array}\right] \\
& =\left[\begin{array}{ccccc}
I_{l}-\mathrm{CB} \Gamma & 0 & 0 & \cdots & 0 \\
-\mathrm{CA}_{3} B \Gamma & I_{l}-\mathrm{CB} \Gamma & 0 & \cdots & 0 \\
-\mathrm{CA}_{3}^{2} B \Gamma & -\mathrm{CA}_{3} B \Gamma & I_{l}-\mathrm{CB} \Gamma & \ddots & \vdots \\
\vdots & \ddots & \ddots & \ddots & 0 \\
-\mathrm{CA}_{3}^{N_{1}-1} B \Gamma & \cdots & -\mathrm{CA}_{3}^{2} B \Gamma & -\mathrm{CA}_{3} B \Gamma & I_{l}-\mathrm{CB} \Gamma
\end{array}\right] .
\end{aligned}
$$

From (57) and Property 2, we can know $\rho\left(\psi_{1} \bar{\phi}_{2}+\psi_{2}\right)=\rho\left(I_{l}-\mathrm{CB} \Gamma\right)$. Consequently, $\rho\left(\psi_{1} \bar{\phi}_{2}+\psi_{2}\right)<1$ can be replaced by a more convenient condition $\rho\left(I_{l}-\mathrm{CB} \Gamma\right)<1$ without using any knowledge on the system matrix $A_{3}$ and the interval length $N_{1}$ in the 2-D LDFFM with input delay (37).

Remark 8. In Remark 7, using the estimated information on $C$ and $B$, the learning gain matrix $\Gamma$ of $\rho\left(I_{l}-\mathrm{CB} \Gamma\right)<1$ can be decided by using the LMI technique. Therefore, a similar Remark 4 can be obtained.

Remark 9. When the RSA on dynamical direction $n_{2}$ is applied to 2-D LDFFM with input delay (37) and the ILC law (38), the same ILC result with Theorem 2 can be obtained, the proof process of which is shown in Appendix 7.3.

Remark 10. It is noted that 2-D LDFFM (1) and (37) contain 2-D Attasi model as a special case, i.e.,
$A_{2}=-A_{1} A_{3}=-A_{3} A_{1}$. And the 2-D Roesser model under some specified coefficient matrix requirements can also be converted into 2-D LDFFM [29]. Consequently, depending on the ILC results obtained from 2-D LDFFM (1) and (37), it is easy to extend 2-D Roesser model and 2-D Attasi model with input delay.

\section{Illustrative Examples}

To show the effectiveness and feasibility of the proposed ILC algorithms (9) and (38) for the 2-D LDFFM with a direct transmission from inputs to outputs (1) and 2-D LDFFM with input delay (37), two simulation examples are introduced in this section.

Example 1. Consider the following 2-D LDFFM with a direct transmission from inputs to outputs over dynamical region $\left\{n_{1}\right\}_{0}^{20}$ and $\left\{n_{2}\right\}_{0}^{20}$ : 


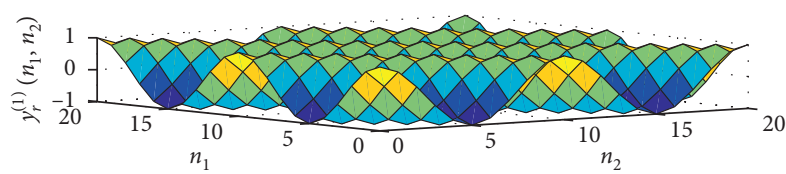

(a)

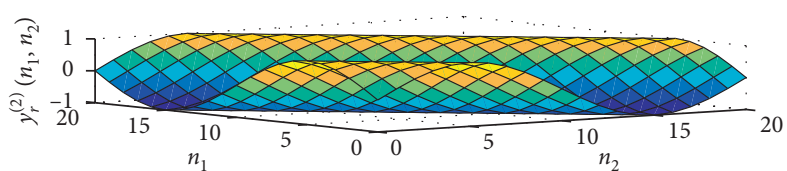

(b)

FIGURE 1: Reference surfaces $y_{r}^{(1)}\left(n_{1}, n_{2}\right)$ and $y_{r}^{(2)}\left(n_{1}, n_{2}\right)$ for $\left\{n_{1}\right\}_{0}^{20}$ and $\left\{n_{2}\right\}_{0}^{20}$.

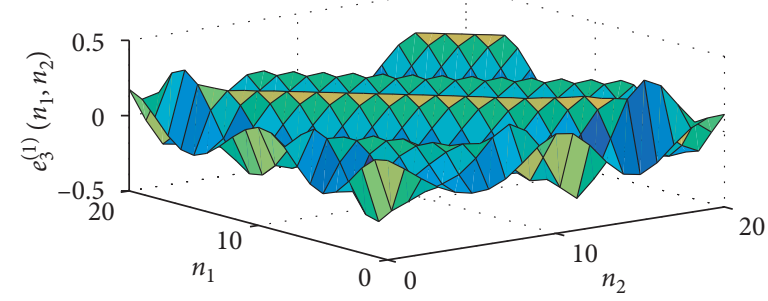

(a)

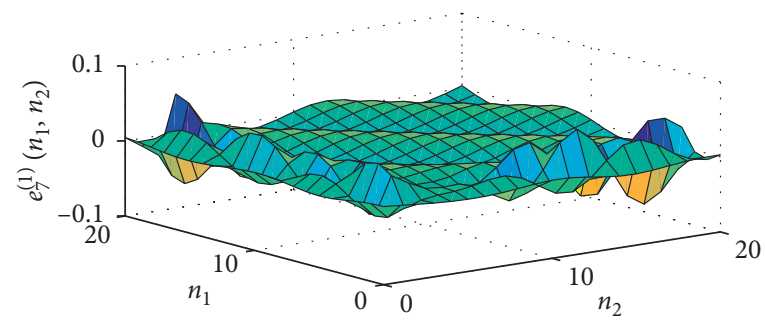

(c)

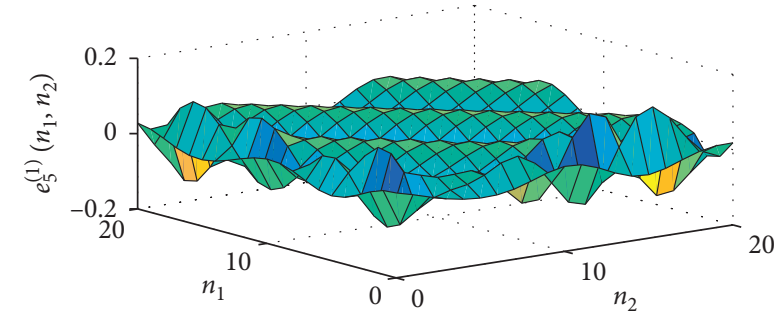

(b)

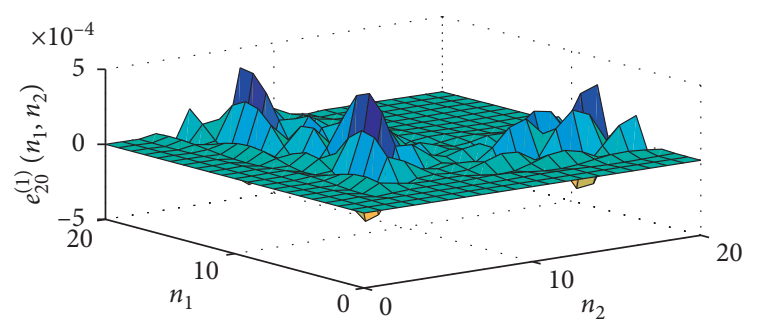

(d)

FIgURE 2: Tracking error surface $e_{k}^{(1)}\left(n_{1}, n_{2}\right)$ at $k=3,5,7,20$ by using the ILC law (9).

$$
\begin{aligned}
{\left[\begin{array}{l}
x_{k}^{(1)}\left(n_{1}+1, n_{2}+1\right) \\
x_{k}^{(2)}\left(n_{1}+1, n_{2}+1\right)
\end{array}\right]=} & {\left[\begin{array}{ll}
0.01 & 0.01 \\
0.01 & 0.03
\end{array}\right]\left[\begin{array}{l}
x_{k}^{(1)}\left(n_{1}+1, n_{2}\right) \\
x_{k}^{(2)}\left(n_{1}+1, n_{2}\right)
\end{array}\right]+\left[\begin{array}{ll}
0.01 & 0.02 \\
0.01 & 0.04
\end{array}\right]\left[\begin{array}{l}
x_{k}^{(1)}\left(n_{1}, n_{2}\right) \\
x_{k}^{(2)}\left(n_{1}, n_{2}\right)
\end{array}\right] } \\
& +\left[\begin{array}{cc}
0.01 & 0.02 \\
0.01 & 0.3
\end{array}\right]\left[\begin{array}{l}
x_{k}^{(1)}\left(n_{1}, n_{2}+1\right) \\
x_{k}^{(2)}\left(n_{1}, n_{2}+1\right)
\end{array}\right]+\left[\begin{array}{cc}
1 & 0.2 \\
0.3 & 0.5
\end{array}\right]\left[\begin{array}{l}
u_{k}^{(1)}\left(n_{1}, n_{2}\right) \\
u_{k}^{(2)}\left(n_{1}, n_{2}\right)
\end{array}\right], \\
{\left[\begin{array}{l}
y_{k}^{(1)}\left(n_{1}, n_{2}\right) \\
y_{k}^{(2)}\left(n_{1}, n_{2}\right)
\end{array}\right]=} & {\left[\begin{array}{cc}
1 & 0.2 \\
-0.2 & 1
\end{array}\right]\left[\begin{array}{l}
x_{k}^{(1)}\left(n_{1}, n_{2}\right) \\
x_{k}^{(1)}\left(n_{1}, n_{2}\right)
\end{array}\right]+\left[\begin{array}{ll}
2 & 0 \\
0 & 2
\end{array}\right]\left[\begin{array}{l}
u_{k}^{(1)}\left(n_{1}, n_{2}\right) \\
u_{k}^{(2)}\left(n_{1}, n_{2}\right)
\end{array}\right], }
\end{aligned}
$$

which is required to repetitively track a desired reference surface $\quad y_{r}\left(n_{1}, n_{2}\right)=\left[\cos \left(2 \pi\left(n_{1}+n_{2}\right)\right) / 10 \sin \left(\pi\left(n_{1}+\right.\right.\right.$ $\left.\left.\left.n_{2}\right)\right) / 10\right]^{T}$ for $\left\{n_{1}\right\}_{0}^{20}$ and $\left\{n_{2}\right\}_{0}^{20}$ shown in Figure 1. Under Assumption 2, the boundary states of 2-D LDFFM (58) are given as $x_{k}\left(0, n_{2}\right)=\left[0.1 \sin \left(n_{2}\right)\right]^{T},\left\{n_{2}\right\}_{1}^{20}$, and $x_{k}\left(n_{1}, 0\right)=$

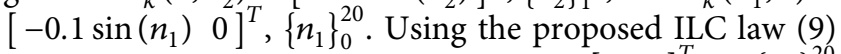
with the initial control input $u_{0}\left(n_{1}, n_{2}\right)=\left[\begin{array}{ll}0 & 0\end{array}\right]^{T}$ for $\left\{n_{1}\right\}_{0}^{20}$ and $\left\{n_{2}\right\}_{0}^{20}$, the gain matrix $\Gamma$ is selected as $\Gamma=\operatorname{diag}\left\{\begin{array}{ll}0.3 & 0.3\end{array}\right\}$, which satisfies the convergence condition $\rho\left(I_{l}-D \Gamma\right)=0.6<1$ in Theorem 1 . In order to evaluate the accuracy of ILC tracking, the following maximum absolute tracking error index

$$
\operatorname{MATE}_{k}^{(h)}=\max _{\left\{n_{1}\right\}_{0}^{20}} \max _{\left\{n_{2}\right\}_{0}^{20}}\left|y_{r}^{(h)}\left(n_{1}, n_{2}\right)-y_{k}^{(h)}\left(n_{1}, n_{2}\right)\right|, \quad\{h\}_{1}^{2},
$$

is used. As a result, the ILC tracking error surfaces $e_{k}^{(1)}\left(n_{1}, n_{2}\right)$ at $k=3,5,7,25$, and $e_{k}^{(2)}\left(n_{1}, n_{2}\right)$ at $k=4,6,8,24$ are depicted in Figures 2 and 3, respectively. Also, Figure 4 presents the profiles of the ILC tracking error indexes $\operatorname{MATE}_{k}^{(1)}$ and $\mathrm{MATE}_{k}^{(2)}$ in iteration domain $k$ by utilizing the ILC law (9). Obviously, it is observed from Figures 2-4 that the convergent effect of the ILC law (9) against the iterationinvariant boundary states is illustrated. 


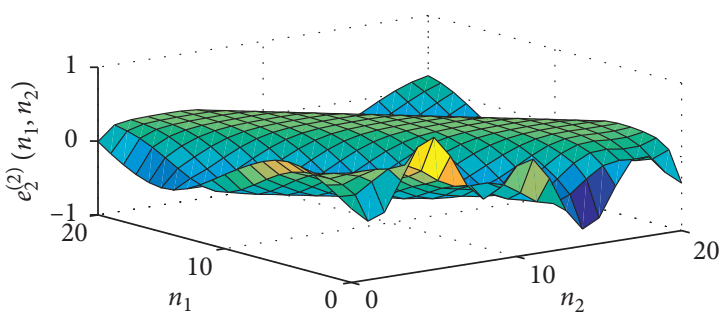

(a)

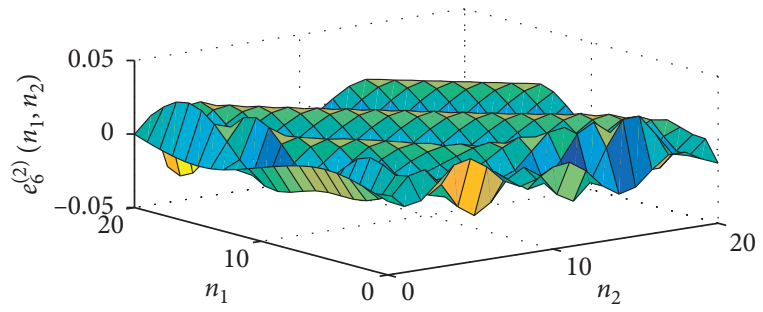

(c)

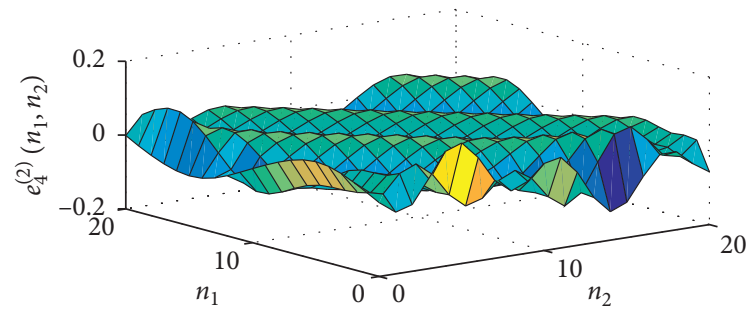

(b)

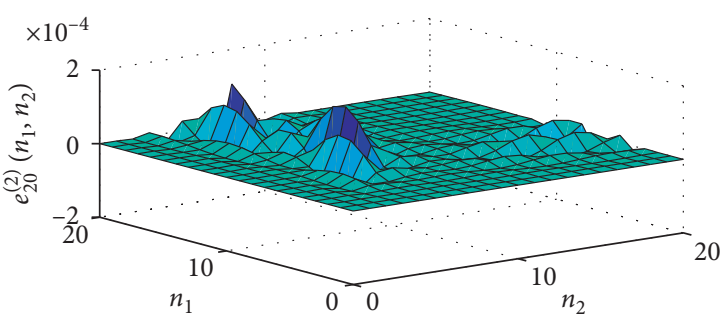

(d)

Figure 3: Tracking error surface $e_{k}^{(2)}\left(n_{1}, n_{2}\right)$ at $k=2,4,6,20$ by using the ILC law (9).

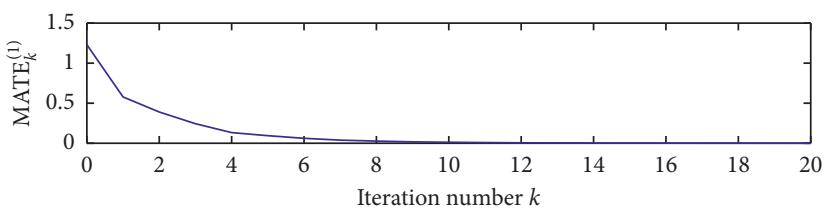

(a)

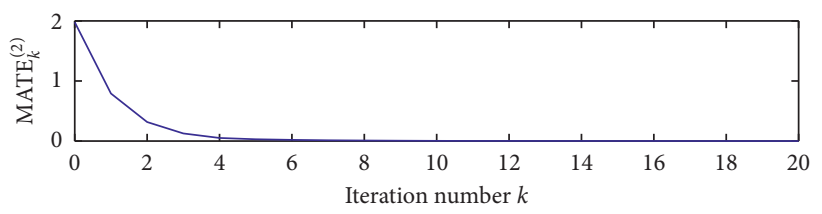

(b)

FIgURE 4: Tracking error indexes $\operatorname{MATE}_{k}^{(1)}$ and $\operatorname{MATE}_{k}^{(2)}$ with iteration number $k$ by using the ILC law (9).

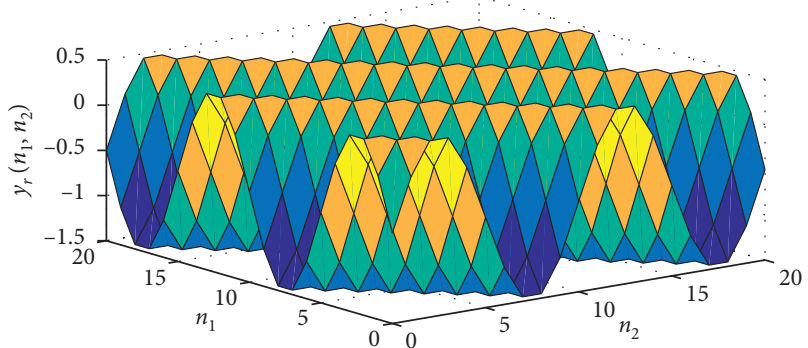

FIgURE 5: Reference surface $y_{r}\left(n_{1}, n_{2}\right)$ for $\left\{n_{1}\right\}_{0}^{20}$ and $\left\{n_{2}\right\}_{0}^{20}$.

Example 2. Some practical thermal processes can be represented by the following partial differential equation (PDE) [31]:

$$
\begin{aligned}
\frac{\partial^{2} s_{k}(w, t)}{\partial w \partial t}= & a_{1} \frac{\partial s_{k}(w, t)}{\partial t}+a_{2} \frac{\partial s_{k}(w, t)}{\partial w}+a_{0} s_{k}(w, t) \\
& +b f_{k}\left(w-d_{w}, t-d_{t}\right)
\end{aligned}
$$

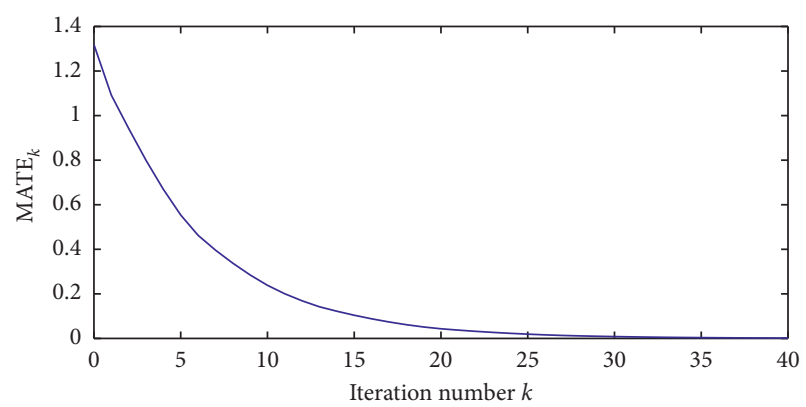

FIgURE 6: Tracking error index $\mathrm{MATE}_{k}$ with iteration number $k$ by using the ILC law (38).

where $d_{w}$ and $d_{t}$, respectively, denote space delay and time delay; $a_{0}, a_{1}, a_{2}$, and $b$ are real coefficients. The repetitive index $k$ implies that the ILC issue is investigated in the example. Through the discretization approximation on (60), system parameters and delay factors of 2-D LDFFM with input delay (37) are given as follows: 


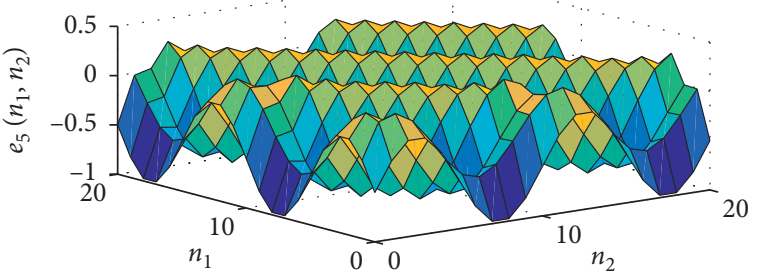

(a)

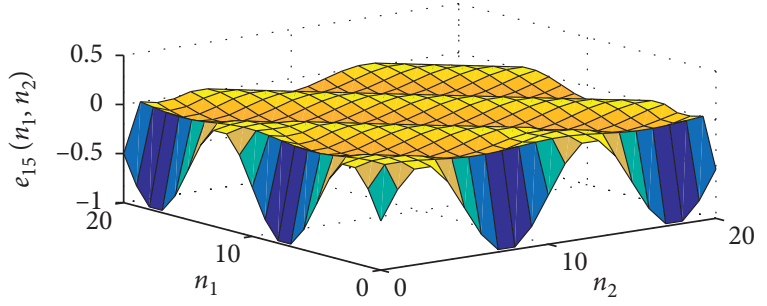

(c)

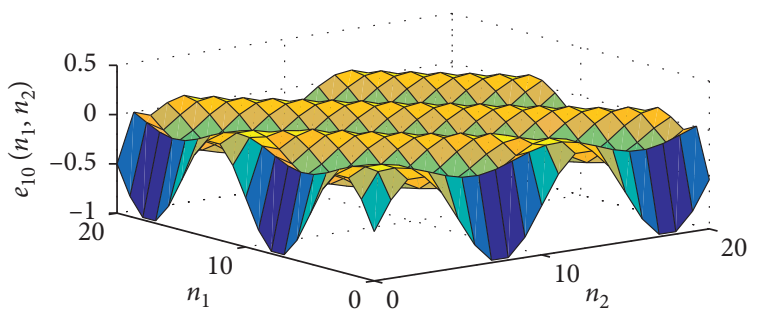

(b)

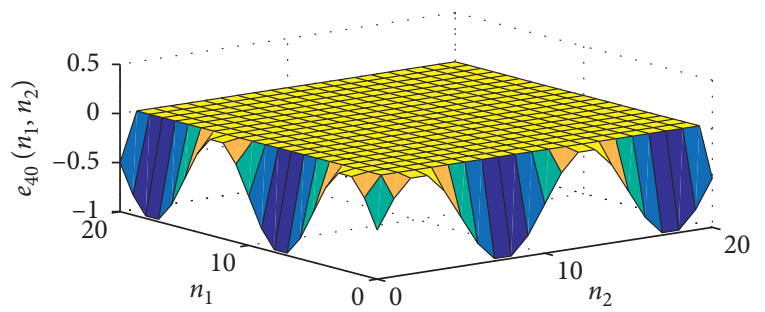

(d)

Figure 7: Tracking error surface $e_{k}\left(n_{1}, n_{2}\right)$ at $k=5,10,15,40$ by using the ILC law (38).

$$
\begin{aligned}
A_{1} & =1+a_{2} \Delta t \\
A_{2} & =a_{0} \Delta w \Delta t-a_{1} \Delta w-a_{2} \Delta t-1, \\
A_{3} & =1+a_{1} \Delta w \\
B & =b \Delta w \Delta t \\
\tau_{1} & =\operatorname{int}\left(\frac{d_{w}}{\Delta w}\right) \\
\tau_{2} & =\operatorname{int}\left(\frac{d_{t}}{\Delta t}\right),
\end{aligned}
$$

where is shown in Figure 5. And the boundary states satisfy $x_{k}\left(0, n_{2}\right)=0.5 \sin \left(2 \pi n_{2} / 10\right),\left\{n_{2}\right\}_{1}^{20}$, and $x_{k}\left(n_{1}, 0\right)=$ $0.5 \sin \left(2 \pi n_{1} / 10\right),\left\{n_{1}\right\}_{0}^{20}$. Under the ILC law (38) with initial control input $u_{0}\left(n_{1}, n_{2}\right)=0$ for $\left\{n_{1}\right\}_{-1}^{18}$ and $\left\{n_{2}\right\}_{-2}^{17}$, let the learning gain $\Gamma$ be selected as $\Gamma=0.9$ and compute $\rho\left(I_{l}-C B \Gamma\right)=0.7885<1$, which satisfies the convergent condition in Theorem 2 . The maximum absolute tracking error index $\mathrm{MATE}_{k}$ is adopted to evaluate the ILC tracking performance, which is given as follows:

$$
\mathrm{MATE}_{k}=\max _{\left\{n_{1}\right\}_{1}^{20}} \max _{\left\{n_{2}\right\}_{1}^{20}}\left|y_{r}\left(n_{1}, n_{2}\right)-y_{k}\left(n_{1}, n_{2}\right)\right| \text {. }
$$

And Figure 6 displays the profile of $\mathrm{MATE}_{k}$ with iteration number $k$ by using the ILC law (38). The tracking error surface $e_{k}\left(n_{1}, n_{2}\right)$ at $k=5,10,15,40$ is shown in Figure 7. Apparently, perfect tracking on repetitive reference surface can be observed from Figures 6 and 7.

\section{Conclusions}

To date, ILC tracking issues for 2-D LDFFM with a direct transmission from inputs to outputs and with input delay are first concerned in this paper. Different from existing ILC results for 2-D LDFFM, a 3-D analysis approach is first applied to the convergent property of the proposed ILC law, which supplies a new tool for follow-up ILC development for 2-D systems. Additionally, the LMI technique is used to compute the learning gain. In our future work, reinforcement learning (RL) [35-38] will be incorporated into the ILC designs for 2-D dynamical systems such that more ILC results are made.

\section{Appendix}

Proof of Lemma 1. The solution to $\theta_{k+1}\left(n_{1}, n_{2}\right)=\alpha_{1} \theta_{k}\left(n_{1}, n_{2}\right)+\alpha_{2} \delta_{k}\left(n_{1}, n_{2}\right)$ is given as follows:

$$
\theta_{k}\left(n_{1}, n_{2}\right)=\alpha_{1}^{k-1} \theta_{0}\left(n_{1}, n_{2}\right)+\sum_{i=0}^{k-1} \alpha_{1}^{k-i-1} \alpha_{2} \delta_{i}\left(n_{1}, n_{2}\right)
$$

Then, taking the limit on both sides of (A.1), there is

$$
\begin{aligned}
\lim _{k \longrightarrow+\infty} \theta_{k}\left(n_{1}, n_{2}\right)= & \lim _{k \longrightarrow+\infty} \alpha_{1}^{k-1} \theta_{0}\left(n_{1}, n_{2}\right) \\
& +\lim _{k \longrightarrow+\infty} \sum_{i=0}^{k-1} \alpha_{1}^{k-i-1} \alpha_{2} \delta_{i}\left(n_{1}, n_{2}\right) .
\end{aligned}
$$

Under $\lim \theta_{k}\left(n_{1}, n_{2}\right)=0$ in Lemma 1 , if $\rho\left(\alpha_{1}\right)<1$ holds, we obtain

$$
\lim _{k \longrightarrow+\infty} \theta_{k}\left(n_{1}, n_{2}\right)=0
$$

Lemma 1 is completed. 
Proof of Theorem 1 by using the RSA. Let

$$
\begin{gathered}
\delta \bar{X}_{k}\left(n_{1}\right)=\left[\begin{array}{llll}
\delta x_{k}^{T}\left(n_{1}, 1\right) & \delta x_{k}^{T}\left(n_{1}, 2\right) & \cdots & \delta x_{k}^{T}\left(n_{1}, N_{2}\right)
\end{array}\right]^{T}, \\
\bar{E}_{k}\left(n_{1}\right)=\left[\begin{array}{llll}
e_{k}^{T}\left(n_{1}, 0\right) & e_{k}^{T}\left(n_{1}, 1\right) & \cdots & e_{k}^{T}\left(n_{1}, N_{2}\right)
\end{array}\right]^{T} .
\end{gathered}
$$

From (A.4) and (A.5), using the RSA on vertical direction $n_{2}$, for $\left\{n_{1}\right\}_{0}^{N_{1}-1}$, (14) can be rewritten as

$$
\begin{aligned}
\widehat{\Phi}_{1} \delta \bar{X}_{k}\left(n_{1}+1\right)= & \widehat{\Phi}_{2} \delta \bar{X}_{k}\left(n_{1}\right)+\widehat{\Phi}_{3} \bar{E}_{k}\left(n_{1}\right)+\widehat{A}_{2} \delta x_{k}\left(n_{1}, 0\right) \\
& +\widehat{A}_{1} \delta x_{k}\left(n_{1}+1,0\right),
\end{aligned}
$$

where

$$
\begin{aligned}
& \widehat{\Phi}_{1}=\left[\begin{array}{ccccc}
I_{p} & 0 & 0 & \cdots & 0 \\
-A_{1} & I_{p} & 0 & \cdots & 0 \\
0 & -A_{1} & I_{p} & \ddots & \vdots \\
\vdots & \ddots & \ddots & \ddots & 0 \\
0 & \cdots & 0 & -A_{1} & I_{p}
\end{array}\right] \in R^{p N_{2} \times p N_{2}}, \\
& \widehat{\Phi}_{2}=\left[\begin{array}{ccccc}
A_{3} & 0 & 0 & \cdots & 0 \\
A_{2} & A_{3} & 0 & \cdots & 0 \\
0 & A_{2} & A_{3} & \ddots & \vdots \\
\vdots & \ddots & \ddots & \ddots & 0 \\
0 & \cdots & 0 & A_{2} & A_{3}
\end{array}\right] \in R^{p N_{2} \times p N_{2}}, \\
& \widehat{\Phi}_{3}=\left[\begin{array}{ccccc}
B \Gamma & 0 & 0 & \cdots & 0 \\
0 & B \Gamma & \ddots & \ddots & \vdots \\
0 & 0 & B \Gamma & 0 & 0 \\
\vdots & \ddots & \ddots & \ddots & 0 \\
0 & \cdots & 0 & 0 & B \Gamma
\end{array}\right] \in R^{p N_{2} \times p\left(N_{2}+1\right)} \text {, } \\
& \widehat{A}_{1}=\left[\begin{array}{c}
A_{1} \\
0 \\
\vdots \\
0
\end{array}\right] \in R^{p N_{2} \times p} \text {, } \\
& \widehat{A}_{2}=\left[\begin{array}{c}
A_{2} \\
0 \\
\vdots \\
0
\end{array}\right] \in R^{p N_{2} \times p} \text {. }
\end{aligned}
$$

Since $\widehat{\Phi}_{1}$ is a nonsingular matrix, premultiplying $\widehat{\Phi}_{1}^{-1}$ on both sides of (A.6), we get

$$
\begin{aligned}
\delta \bar{X}_{k}\left(n_{1}+1\right)= & \widehat{\Phi}_{1}^{-1} \widehat{\Phi}_{2} \delta \bar{X}_{k}\left(n_{1}\right)+\widehat{\Phi}_{1}^{-1} \widehat{\Phi}_{3} \bar{E}_{k}\left(n_{1}\right) \\
& +\widehat{\Phi}_{1}^{-1} \widehat{A}_{2} \delta x_{k}\left(n_{1}, 0\right)+\Phi_{1}^{-1} \widehat{A}_{1} \delta x_{k}\left(n_{1}+1,0\right)
\end{aligned}
$$

Similarly, (17) can also be reformulated as

$$
\bar{E}_{k+1}\left(n_{1}\right)=\varphi_{1} \delta \bar{X}_{k}\left(n_{1}\right)+\varphi_{2} \bar{E}_{k}\left(n_{1}\right)-\widehat{C} \delta x_{k}\left(n_{1}, 0\right),
$$

where

$$
\begin{aligned}
\varphi_{1} & =\left[\begin{array}{ccccc}
0 & 0 & 0 & \cdots & 0 \\
-C & 0 & 0 & \cdots & 0 \\
0 & -C & 0 & \ddots & 0 \\
\vdots & \ddots & \ddots & \ddots & \vdots \\
0 & \cdots & 0 & -C & 0
\end{array}\right] \in R^{l\left(N_{2}+1\right) \times p N_{2},} \\
\varphi_{2} & =\left[\begin{array}{cccc}
I_{l}-D \Gamma & 0 & \cdots & 0 \\
0 & I_{l}-D \Gamma & \ddots & \vdots \\
\vdots & \ddots & \ddots & 0 \\
0 & \cdots & 0 & I_{l}-D \Gamma
\end{array}\right] \in R^{l\left(N_{2}+1\right) \times l\left(N_{2}+1\right)}, \\
\widehat{C} & =\left[\begin{array}{c}
C \\
0 \\
\vdots \\
0
\end{array}\right] \in R^{l\left(N_{2}+1\right) \times p} .
\end{aligned}
$$

From Assumption 2, we obtain $\delta_{k}\left(n_{1}, 0\right)=x_{k+1}\left(n_{1}, 0\right)-$ $x_{k}\left(n_{1}, 0\right)=0$ for $\left\{n_{1}\right\}_{0}^{N_{1}}$. Thus, (A.8) and (A.9) can be expressed as

$$
\left\{\begin{array}{l}
\delta \bar{X}_{k}\left(n_{1}+1\right)=\widehat{\Phi}_{1}^{-1} \widehat{\Phi}_{2} \delta \bar{X}_{k}\left(n_{1}\right)+\widehat{\Phi}_{1}^{-1} \widehat{\Phi}_{3} \bar{E}_{k}\left(n_{1}\right) \\
\bar{E}_{k+1}\left(n_{1}\right)=\varphi_{1} \delta \bar{X}_{k}\left(n_{1}\right)+\varphi_{2} \bar{E}_{k}\left(n_{1}\right)
\end{array}\right.
$$

where $\left\{n_{1}\right\}_{0}^{N_{1}-1}$. In (A.11), according to Assumption 2, we deduce

$$
\delta \bar{X}_{k}(0)=\left[\begin{array}{lllll}
\delta x_{k}^{T}(0,1) & \delta x_{k}^{T}(0,2) & \cdots & \delta x_{k}^{T}\left(0, N_{2}\right)
\end{array}\right]^{T}=0,
$$

and $E_{0}\left(n_{1}\right)$ is bounded for $\left\{n_{1}\right\}_{0}^{N_{1}-1}$. Using Lemma 4 in [18] and Property 2, if $\rho\left(I_{l}-D \Gamma\right)<1$, there is

$$
\left\{\begin{array}{l}
\lim _{k \longrightarrow+\infty} \delta \bar{X}_{k}\left(n_{1}\right)=0,\left\{n_{1}\right\}_{1}^{N_{1}}, \\
\lim _{k \longrightarrow+\infty} \bar{E}_{k}\left(n_{1}\right)=0,\left\{n_{1}\right\}_{0}^{N_{1}-1} .
\end{array}\right.
$$

In addition, taking $n_{1}=N_{1}$ in (17), we have

$$
e_{k+1}\left(N_{1}, n_{2}\right)=-C \delta x_{k}\left(N_{1}, n_{2}\right)+\left(I_{l}-D \Gamma\right) e_{k}\left(N_{1}, n_{2}\right) \text {. }
$$

From (A.13) and $\delta x_{k}\left(N_{1}, 0\right)=0$, using Lemma 1, if $\rho\left(I_{l}-D \Gamma\right)<1$, it yields

$$
\lim _{k \longrightarrow+\infty} e_{k}\left(N_{1}, n_{2}\right)=0, \quad\left\{n_{2}\right\}_{0}^{N_{2}} .
$$

From (A.13) and (A.15) and the definition of $\bar{E}_{k}\left(n_{1}\right)$ in (A.5), we obtain

$$
\lim _{k \rightarrow+\infty} e_{k}\left(n_{1}, n_{2}\right)=0, \quad\left\{n_{1}\right\}_{0}^{N_{1}},\left\{n_{2}\right\}_{0}^{N_{2}} .
$$


Theorem 1 is completed.

Proof of Theorem 2 by using the RSA. For $\left\{n_{1}\right\}_{0}^{N_{1}}$ and $\left\{n_{2}\right\}_{0}^{N_{2}}$, define

$$
\delta \tilde{x}_{k}\left(n_{1}+1, n_{2}\right)=x_{k+1}\left(n_{1}, n_{2}\right)-x_{k}\left(n_{1}, n_{2}\right) .
$$

From (A.17), there is

$$
\begin{aligned}
\delta \tilde{x}_{k}\left(n_{1}+1, n_{2}+1\right)= & x_{k+1}\left(n_{1}, n_{2}+1\right)-x_{k}\left(n_{1}, n_{2}+1\right) \\
= & A_{1} \delta \widetilde{x}_{k}\left(n_{1}+1, n_{2}\right)+A_{2} \delta \tilde{x}_{k}\left(n_{1}, n_{2}\right) \\
& +A_{3} \delta \tilde{x}_{k}\left(n_{1}, n_{2}+1\right) \\
& +B\left[u_{k+1}\left(n_{1}-\tau_{1}-1, n_{2}-\tau_{2}\right)\right. \\
& \left.-u_{k}\left(n_{1}-\tau_{1}-1, n_{2}-\tau_{2}\right)\right],
\end{aligned}
$$

where $\left\{n_{1}\right\}_{1}^{N_{1}}$ and $\left\{n_{2}\right\}_{0}^{N_{2}-1}$. Inserting the ILC law (38) into (A.18), it generates

$$
\begin{aligned}
\delta \tilde{x}_{k}\left(n_{1}+1, n_{2}+1\right)= & A_{1} \delta \tilde{x}_{k}\left(n_{1}+1, n_{2}\right)+A_{2} \delta \tilde{x}_{k}\left(n_{1}, n_{2}\right) \\
& +A_{3} \delta \widetilde{x}_{k}\left(n_{1}, n_{2}+1\right) \\
& +B \Gamma e_{k}\left(n_{1}, n_{2}+1\right) .
\end{aligned}
$$

On the contrary, according to $e_{k}\left(n_{1}, n_{2}\right)=y_{r}\left(n_{1}, n_{2}\right)-$ $y_{k}\left(n_{1}, n_{2}\right)$ and using (A.17), we have

$$
\begin{aligned}
e_{k+1} & \left(n_{1}, n_{2}+1\right)-e_{k}\left(n_{1}, n_{2}+1\right) \\
= & y_{r}\left(n_{1}, n_{2}+1\right)-y_{k+1}\left(n_{1}, n_{2}+1\right)-y_{r}\left(n_{1}, n_{2}+1\right) \\
& +y_{k}\left(n_{1}, n_{2}+1\right) \\
= & -C\left[x_{k+1}\left(n_{1}, n_{2}+1\right)-x_{k}\left(n_{1}, n_{2}+1\right)\right] \\
= & -C \delta \tilde{x}_{k}\left(n_{1}+1, n_{2}+1\right),
\end{aligned}
$$

where $\left\{n_{1}\right\}_{0}^{N_{1}}$ and $\left\{n_{2}\right\}_{0}^{N_{2}-1}$. Let

$$
\begin{aligned}
& \delta \widetilde{X}_{k}\left(n_{1}\right)=\left[\begin{array}{llll}
\delta x_{k}^{T}\left(n_{1}, 1\right) & \delta x_{k}^{T}\left(n_{1}, 2\right) & \cdots & \delta x_{k}^{T}\left(n_{1}, N_{2}\right)
\end{array}\right]^{T}, \\
& \widetilde{E}_{k}\left(n_{1}\right)=\left[\begin{array}{llll}
e_{k}^{T}\left(n_{1}, 1\right) & e_{k}^{T}\left(n_{1}, 2\right) & \cdots & e_{k}^{T}\left(n_{1}, N_{2}\right)
\end{array}\right]^{T},
\end{aligned}
$$

where $\left\{n_{1}\right\}_{1}^{N_{1}}$. From (A.21) and (A.22), using the RSA on vertical dynamical direction $n_{2}$, (A.19) can be rewritten as

$$
\begin{aligned}
\widetilde{\Phi}_{1} \delta \widetilde{X}_{k}\left(n_{1}+1\right)= & \widetilde{\Phi}_{2} \delta \widetilde{X}_{k}\left(n_{1}\right)+\widetilde{\Phi}_{3} \widetilde{E}_{k}\left(n_{1}\right)+\widetilde{A}_{2} \delta \widetilde{x}_{k}\left(n_{1}, 0\right) \\
& +\widetilde{A}_{1} \delta \widetilde{x}_{k}\left(n_{1}+1,0\right), \quad\left\{n_{1}\right\}_{0}^{N_{1}-1},
\end{aligned}
$$

where

$$
\begin{aligned}
& \widetilde{\Phi}_{1}=\left[\begin{array}{ccccc}
I_{p} & 0 & 0 & \cdots & 0 \\
-A_{1} & I_{p} & 0 & \ddots & \vdots \\
0 & -A_{1} & I_{p} & \ddots & 0 \\
\vdots & \ddots & \ddots & \ddots & 0 \\
0 & \cdots & 0 & -A_{1} & I_{p}
\end{array}\right] \in R^{p N_{2} \times p N_{2}}, \\
& \widetilde{\Phi}_{2}=\left[\begin{array}{ccccc}
A_{3} & 0 & 0 & \cdots & 0 \\
A_{2} & A_{3} & 0 & \ddots & \vdots \\
0 & A_{2} & A_{3} & \ddots & 0 \\
\vdots & \ddots & \ddots & \ddots & 0 \\
0 & \cdots & 0 & A_{2} & A_{3}
\end{array}\right] \in R^{p N_{2} \times p N_{2}}, \\
& \widetilde{\Phi}_{3}=\left[\begin{array}{ccccc}
B \Gamma & 0 & 0 & \cdots & 0 \\
0 & B \Gamma & 0 & \ddots & \vdots \\
0 & 0 & B \Gamma & \ddots & 0 \\
\vdots & \ddots & \ddots & \ddots & 0 \\
0 & \cdots & 0 & 0 & B \Gamma
\end{array}\right] \in R^{p N_{2} \times l N_{2}}, \\
& \widetilde{A}_{1}=\left[\begin{array}{c}
A_{1} \\
0 \\
\vdots \\
0
\end{array}\right] \in R^{p N_{2} \times p} \text {, } \\
& \widetilde{A}_{2}=\left[\begin{array}{c}
A_{2} \\
0 \\
\vdots \\
0
\end{array}\right] \in R^{p N_{2} \times p} \text {. }
\end{aligned}
$$

Premultiplying $\widetilde{\Phi}_{1}^{-1}$ on both sides of (A.23), we get

$$
\begin{aligned}
\delta \widetilde{X}_{k}\left(n_{1}+1\right)= & \widetilde{\Phi}_{1}^{-1} \widetilde{\Phi}_{2} \delta \widetilde{X}_{k}\left(n_{1}\right)+\widetilde{\Phi}_{1}^{-1} \widetilde{\Phi}_{3} \widetilde{E}_{k}\left(n_{1}\right) \\
& +\widetilde{\Phi}_{1}^{-1} \widetilde{A}_{2} \delta \widetilde{x}_{k}\left(n_{1}, 0\right)+\widetilde{\Phi}_{1}^{-1} \widetilde{A}_{1} \delta \widetilde{x}_{k}\left(n_{1}+1,0\right) .
\end{aligned}
$$

Similarly, (A.20) can also be reformulated as

$$
\widetilde{E}_{k+1}\left(n_{1}\right)=\widetilde{\varphi}_{1} \delta \widetilde{X}_{k}\left(n_{1}+1\right)+\widetilde{\varphi}_{2} \widetilde{E}_{k}\left(n_{1}\right),
$$


where

$$
\begin{aligned}
\widetilde{\varphi}_{1} & =\left[\begin{array}{cccc}
-C & 0 & \cdots & 0 \\
0 & -C & \ddots & \vdots \\
\vdots & \ddots & \ddots & 0 \\
0 & \cdots & 0 & -C
\end{array}\right] \in R^{l N_{2} \times p N_{2}}, \\
\widetilde{\varphi}_{2} & =\left[\begin{array}{cccc}
I_{l} & 0 & \cdots & 0 \\
0 & I_{l} & \ddots & \vdots \\
\vdots & \ddots & \ddots & 0 \\
0 & \cdots & 0 & I_{l}
\end{array}\right] \in R^{l N_{2} \times l N_{2}} .
\end{aligned}
$$

Substituting (A.25) into (A.26), consider $\delta \tilde{x}_{k}\left(n_{1}+1,0\right)=x_{k+1}\left(n_{1}, 0\right)-x_{k}\left(n_{1}, 0\right)=0$ for $\left\{n_{1}\right\}_{0}^{N_{1}}$. Thus, (A.25) and (A.26) can be reformulated as

$$
\left\{\begin{array}{l}
\delta \widetilde{X}_{k}\left(n_{1}+1\right)=\widetilde{\Phi}_{1}^{-1} \widetilde{\Phi}_{2} \delta \widetilde{X}_{k}\left(n_{1}\right)+\widetilde{\Phi}_{1}^{-1} \widetilde{\Phi}_{3} \widetilde{E}_{k}\left(n_{1}\right), \\
\widetilde{E}_{k+1}\left(n_{1}\right)=\widetilde{\varphi}_{1} \widetilde{\Phi}_{1}^{-1} \widetilde{\Phi}_{2} \delta \widetilde{X}_{k}\left(n_{1}\right)+\left(\widetilde{\varphi}_{1} \widetilde{\Phi}_{1}^{-1} \widetilde{\Phi}_{3}+\widetilde{\varphi}_{2}\right) \widetilde{E}_{k}\left(n_{1}\right),
\end{array}\right.
$$

where $\left\{n_{1}\right\}_{1}^{N_{2}}$. Based on Assumption 2, the initial condition in (A.28) is given as

$$
\delta \widetilde{X}_{k}(1)=\left[\begin{array}{lllll}
\delta \widetilde{x}_{k}^{T}(1,1) & \delta \widetilde{x}_{k}^{T}(1,2) & \cdots & \delta \widetilde{x}_{k}^{T}\left(1, N_{2}\right)
\end{array}\right]^{T}=0,
$$

and $\widetilde{E}_{0}\left(n_{1}\right)$ is bounded for $\left\{n_{1}\right\}_{1}^{N_{1}}$. Using Lemma 4 in [18], if $\rho\left(\widetilde{\varphi}_{1} \widetilde{\Phi}_{1} \widetilde{\Phi}_{3}+\widetilde{\varphi}_{2}\right)<1 \quad$ (equivalently, $\quad \rho\left(I_{l}-\mathrm{CB} \Gamma\right)<1$, see Property 2$)$, there is

$$
\lim _{k \longrightarrow+\infty} \widetilde{E}_{k}\left(n_{1}\right)=0, \quad\left\{n_{1}\right\}_{1}^{N_{1}}
$$

From (A.22), it yields

$$
\lim _{k \longrightarrow+\infty} e_{k}\left(n_{1}, n_{2}\right)=0, \quad\left\{n_{1}\right\}_{1}^{N_{1}},\left\{n_{2}\right\}_{1}^{N_{2}}
$$

Theorem 2 is completed.

\section{Data Availability}

All the data have been included in the paper; therefore, there are no other data available.

\section{Conflicts of Interest}

The authors declare that there are no conflicts of interest.

\section{Acknowledgments}

This work was supported in part by the National Natural Science Foundation of China (Grant nos. 61903096, 61573385, and 61703444), Science and Technology Program of Guangzhou (Grant no. 201904010475), Professorial and Doctoral Scientific Research Foundation of Huizhou University (Grant no. 2020JB017), and Huizhou Science and Technology Plan Project (Grant no. 2020SD0406034).

\section{References}

[1] E. Rimon and A. Stappen, "Immobilizing 2-D serial chains in form-closure grasps," IEEE Transactions on Robotics, vol. 28, no. 1, pp. 32-43, 2012.

[2] Z. Wei, M. J. Wang, Y. J. Cai, and L. Wang, "Milling force prediction for ball-end milling of 3D curved surfaces," Journal of Mechanical Engineering, vol. 49, no. 01, pp. 178-184, 2013.

[3] Y. Q. Luo, Z. D. Wang, G. L. Liang, and F. E. Alsaadi, " $H_{\infty}$ control for 2-D fuzzy systems with interval time-varying delays and missing measurements," IEEE Transactions on Cybernetics, vol. 47, no. 2, pp. 365-377, 2017.

[4] C. K. Ahn, L. Wu, and P. Shi, "Stochastic stability analysis for 2-D Roesser systems with multiplicative noise," Automatica, vol. 69, pp. 356-363, 2016.

[5] L. Wu and H. Gao, "Sliding mode control of two-dimensional systems in Roesser model," IET Control Theory \& Applications, vol. 2, no. 4, pp. 352-364, 2008.

[6] D. A. Bristow, M. Tharayil, and A. G. Alleyne, "A survey of iterative learning control," IEEE Control Systems Magazine, vol. 26, no. 3, pp. 96-114, 2006.

[7] J.-X. Xu, "A survey on iterative learning control for nonlinear systems," International Journal of Control, vol. 84, no. 7, pp. 1275-1294, 2011.

[8] D. Shen and X. Li, "A survey on iterative learning control with randomly varying trial lengths: model, synthesis, and convergence analysis," Annual Reviews in Control, vol. 48, pp. 89-102, 2019.

[9] M. Yu and S. Chai, "A survey on high-order internal model based iterative learning control," IEEE Access, vol. 7, pp. 127024-127031, 2019.

[10] D. Meng, "Convergence conditions for solving robust iterative learning control problems under nonrepetitive model uncertainties," IEEE Transactions on Neural Networks and Learning Systems, vol. 30, no. 6, pp. 1908-1919, 2019.

[11] X. Bu and Z. Hou, "Adaptive iterative learning control for linear systems with binary-valued observations," IEEE Transactions on Neural Networks and Learning Systems, vol. 29, no. 1, pp. 232-237, 2018.

[12] X. Bu, Q. Yu, Z. Hou, and W. Qian, "Model free adaptive iterative learning consensus tracking control for a class of nonlinear multiagent systems," IEEE Transactions on Systems, Man, and Cybernetics: Systems, vol. 49, no. 4, pp. 677-686, 2019.

[13] X. H. Bu, P. P. Zhu, Z. S. Hou, and J. Q. Liang, "Finite-time consensus for linear multiagent systems using data-driven terminal ILC," IEEE Transactions on Circuits and Systems II: Express Briefs, 2019.

[14] R. H. Chi, Y. Hui, B. Huang, and Z. S. Hou, “Adjacent-agent dynamic linearization-based iterative learning formation control," IEEE Transactions on Cybernetics, 2019.

[15] H. Afkhami, A. Argha, M. Roopaei, and M. A. Nouri, "Optimal iterative learning control method for 2-D systems using 1-D model (WAM) of 2-D systems," World Applied Sciences Journal, vol. 13, pp. 2410-2419, 2011.

[16] M. Roopaei, A. Argha, and P. Karimaghaee, "On the control of 2-D systems by intelligent schemes," World Applied Sciences Journal, vol. 6, pp. 385-392, 2009.

[17] K. Wan and X.-D. Li, "Iterative learning control for twodimensional linear discrete systems with Fornasini-Marchesini model," International Journal of Control, Automation and Systems, vol. 15, no. 4, pp. 1710-1719, 2017.

[18] K. Wan and X. D. Li, "High-order internal model based iterative learning control for 2-D linear FMMI systems with 
iteration-varying trajectory tracking," IEEE Transactions on Systems, Man, Cybernetics: Systems, 2019.

[19] K. Wan and X. D. Li, "Robust iterative learning control of 2-D linear discrete FMMII systems subject to iteration-dependent uncertainties," IEEE Transactions on Systems, Man, Cybernetics: Systems, 2019.

[20] K. Wan, D. Xu, and Y.-s. Wei, "Iterative learning control for 2-D linear discrete Fornasini-Marchesini model with input saturation," International Journal of Systems Science, vol. 51, no. 8, pp. 1482-1494, 2020.

[21] K. Wan and Y. S. Wei, "Adaptive ILC of tracking nonrepetitive trajectory for two dimensional nonlinear discrete time-varying Fornasini-Marchesini systems with iterationvarying boundary states," International Journal of Control, Automation and Systems, in press, 2020.

[22] Q.-Y. Xu, X.-D. Li, and M.-M. Lv, “Adaptive ILC for tracking non-repetitive reference trajectory of 2-D FMM under random boundary condition," International Journal of Control, Automation and Systems, vol. 14, no. 2, pp. 478-485, 2016.

[23] L. G. Wu and Z. D. Wang, Filtering and Control for Classes of Two-Dimensional Systems, Springer, New York, NY, USA, 2014.

[24] S. I. Niculescu, Delay Effects on Stability: A Robust Control Approach, Springer, London, UK, 2001.

[25] J. Liang, Z. Wang, and X. Liu, "Robust state estimation for two-dimensional stochastic time-delay systems with missing measurements and sensor saturation," Multidimensional Systems and Signal Processing, vol. 25, no. 1, pp. 157-177, 2014.

[26] L. Wu, Z. Wang, H. Gao, and C. Wang, "Filtering for uncertain 2-D discrete systems with state delays," Signal Processing, vol. 87, no. 9, pp. 2213-2230, 2007.

[27] J. $\mathrm{Xu}$ and $\mathrm{L}$. Yu, "Delay-dependent $\mathrm{Ho}$ control for 2-D discrete state delay systems in the second FM model," Multidimensional Systems and Signal Processing, vol. 20, no. 4, pp. 333-349, 2009.

[28] A. Tandon and A. Dhawan, "An LMI approach to non-fragile robust optimal guaranteed cost control of uncertain 2-D discrete systems with both state and input delays," Transactions of the Institute of Measurement and Control, vol. 40, no. 3, pp. 785-804, 2018.

[29] T. Kaczorek, Two-dimensional Linear Systems, SpringerVerlag, Heidelberg, Germany, 1985.

[30] R. Thamvichai and T. Bose, "Stability of 2-D periodically shift variant filters," IEEE Transactions on Circuits and Systems II: Analog and Digital Signal Processing, vol. 49, no. 1, pp. 61-64, 2002.

[31] W. Marszalek, "Two-dimensional state-space discrete models for hyperbolic partial differential equations," Applied Mathematical Modelling, vol. 8, no. 1, pp. 11-14, 1984.

[32] R. A. Horn and C. R. Johnson, Matrix Analysis, Cambridge University Press, Cambridge, UK, 1985.

[33] X.-D. Li and J. K. L. Ho, "Further results on iterative learning control with convergence conditions for linear time-variant discrete systems," International Journal of Systems Science, vol. 42, no. 6, pp. 989-996, 2011.

[34] S. Boyd, L. E. Ghaoui, E. Feron, and V. Balakrishnan, Linear Matrix Inequalities in System and Control Theory, SIAM, Philadelphia, PA, USA, 1984.

[35] S. He, H. Fang, M. Zhang, F. Liu, and Z. Ding, "Adaptive optimal control for a class of nonlinear systems: the online policy iteration approach," IEEE Transactions on Neural Networks and Learning Systems, vol. 31, no. 2, pp. 549-558, 2020.
[36] S. He, H. Fang, and M. G. Zhang, "Online policy iterativebased Ho optimization algorithm for a class of nonlinear systems," Information Sciences, vol. 495, pp. 1-13, 2019.

[37] S. He, M. Zhang, H. Fang, F. Liu, X. Luan, and Z. Ding, "Reinforcement learning and adaptive optimization of a class of Markov jump systems with completely unknown dynamic information," Neural Computing and Applications, 2019.

[38] C. Wang, H. Fang, and S. He, "Adaptive optimal controller design for a class of LDI-based neural network systems with input time-delays," Neurocomputing, vol. 385, pp. 292-299, 2020. 